\title{
Crystal Base and a Generalization of the Littlewood-Richardson Rule for the Classical Lie Algebras
}

\section{Toshiki Nakashima}

Department of Mathematical Science, Faculty of Engineering Science, Osaka University, Toyonaka Osaka 560, Japan

\begin{abstract}
We shall give a generalization of the Littlewood-Richardson rule for $U_{q}(\mathrm{~g})$ associated with the classical Lie algebras by use of crystal base. This rule describes explicitly the decomposition of tensor products of given representations.
\end{abstract}

\section{Table of Contents}

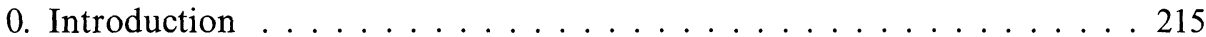

1. Basic Notions of Crystal Base . . . . . . . . . . . . . . . . 217

2. Review of Crystal Graphs . . . . . . . . . . . . . . . . . 218

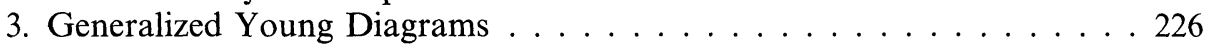

4. Decomposition of $V_{Y} \otimes V_{\square} \ldots \ldots \ldots \ldots \ldots \ldots \ldots \ldots \ldots \ldots \ldots$

5. Decomposition of $V_{Y} \otimes V_{\mathrm{sp}} \ldots \ldots \ldots \ldots \ldots \ldots \ldots \ldots \ldots \ldots$

6. Decomposition of $V_{Y} \otimes V_{W} \ldots \ldots \ldots \ldots \ldots \ldots \ldots \ldots$

Appendix. Relation to the Original Littlewood-Richardson Rule . . . . . 241

\section{Introduction}

In representation theory, it is one of the most fundamental problems to decompose a given representation into the irreducible components. For the Lie algebra $\mathrm{gl}(n)$, we know a very famous rule called the Littlewood-Richardson rule, which gives the irreducible decomposition of the tensor product of two finite-dimensional irreducible representations. There are various generalizations of this rule to other Lie algebras (e.g. cf. [B-Z, L, T]). The purpose of this paper is to give an explicit description of irreducible decomposition of tensor products of finite-dimensional representations of the $q$-analogue of universal enveloping algebra associated with the classical Lie algebras by a new tool "crystal base."

The notion of the $q$-analogue of universal enveloping algebras was introduced by V.G. Drinfeld ([D]) and M. Jimbo ([J]) in 1985 independently. In 1990, the theory of crystal base was constructed by M. Kashiwara ([K1, K2]). Roughly 
speaking, this is the representation theory of the universal enveloping algebra $U_{q}(\mathfrak{g})$ at $q=0$. In the world at $q=0$, various phenomena become much simpler. In particular, the crystal base has a nice property with respect to tensor products of given representations (Theorem 1.1.5 and Proposition 3.2.1).

The crystal base has a colored and oriented graph structure, called a crystal graph (Definition 1.1.7). In [K-N], we describe all the crystal graphs of finitedimensional representations of $U_{q}(\mathfrak{g})\left(\mathfrak{g}=A_{n}, B_{n}, C_{n}, D_{n}\right)$. That gives an explicit parametrization of vertices of the crystal graphs in terms of analogues of semistandard tableaux. The description of the rule of irreducible decompositions depends on the nice property for tensor products and these combinatorial parametrizations of crystal base.

The contents of this paper are as follows. In Sect. 1, the definition and several properties of crystal base are given, in particular, Theorem 1.1.3, Corollary 1.1.4 and Theorem 1.1.5 guarantee the validity of arguments in the later sections. In Sect. 2 , we summarize the results of [K-N], which describes all the crystal graphs of finite-dimensional irreducible representations of $U_{q}(\mathfrak{g})$. First, the crystal graphs of the vector representation and the spin representation are given. Next, the crystal graphs of the fundamental representations are realized in tensor products of the vector representations or the spin representations. Finally, by tensor products of the fundamental weight cases, the crystal graphs in the general case are described. Namely, we embed the crystal graph $B(\lambda)$ of the irreducible module $V(\lambda)$ with highest weight $\lambda$ into $B\left(V_{\square}\right)^{\otimes m}$ or $B(\lambda)$ into $B\left(V_{\square}\right)^{\otimes m} \otimes B\left(V_{\mathrm{sp}}\right)$, where $V_{\square}$ is the vector representation and $V_{\mathrm{sp}}$ is the spin representation. Then we describe $B(\lambda)$ as its image. In Sect. 3, first, we introduce generalized Young diagrams of type $\mathfrak{g}$, which parametrize all finite-dimensional irreducible representations of $U_{q}(\mathfrak{g})$. Next, the following proposition is proved.

Proposition 3.2.1. Let $\lambda$ and $\mu$ be dominant integral weights of g. For $u \in B(\lambda)$ and $v \in B(\mu)$, the following two conditions are equivalent;

(a) $\tilde{e}_{i}(u \otimes v)=0$ for any $i$.

(b) $\tilde{e}_{i} u=0$ and $\tilde{e}_{i}^{\left\langle h_{i}, \lambda\right\rangle+1} v=0$ for any $i$.

By Corollary 1.1.4, we have that $V(\lambda) \otimes V(\mu)$ is decomposed into $V(w t(u \otimes v))$ where $u \otimes v$ ranges over $B(\lambda) \otimes B(\mu)$ satisfying the condition (a). By the condition (b), we know that if $u \otimes v$ is a highest weight element of $B(\lambda) \otimes B(\mu)$, then $u=u_{\lambda}$. In Sects. 4 and 5, the second condition of Proposition 3.2.1(b) is translated in terms of generalized Young diagrams for the special cases $V_{Y} \otimes V_{\square}$ and $V_{Y} \otimes V_{\mathrm{sp}}$ and the rules of decomposition of those representations are given. Those rules are the steps in the procedure for general cases. In Sect. 6, as the consequence of Sects. 1-5, the procedure to obtain the decomposition of $V_{Y} \otimes V_{W}$ is given, where $Y$ and $W$ are generalized Young diagrams of type $\mathfrak{g}$ (Theorem 6.3.1 and Corollary 6.3.2).

The rule of decompositions given in this paper coincides with the corresponding rule in the classical case, namely the case at $q=1$. So, in the appendix, we shall give the 1-1 correspondence between the original Littlewood-Richardson rule and the rule given in this paper for $\operatorname{gl}(n+1)$.

After submitting this paper the author received the preprint "Crystal graphs and Young tableaux" from Littelmann. In that preprint, he also give descriptions of crystal graphs and the Littlewood-Richardson rule for $A_{n}, B_{n}, C_{n}, D_{n}, E_{6}$ and $G_{2}$ in terms of the generalized Young tableaux. 
The author would like to acknowledge M. Kashiwara for valuable advice, A.N. Kirillov for explaining to him several works related to this subject and D.S. McAnally for correcting the manuscript.

\section{Basic Notions of Crystal Base}

In this section, we give the basic notion of crystal base.

1.1. Definitions. Let $\mathfrak{g}$ be a finite-dimensional simple Lie algebra with a Cartan subalgebra $\mathrm{t}$, the set of simple roots $\left\{\alpha_{i} \in \mathrm{t}^{*}\right\}_{i \in I}$ and the set of simple coroots $\left\{h_{i} \in \mathfrak{t}\right\}_{i \in I}$, where I is a finite index set. We take an inner product $\left(\right.$, ) on $\mathrm{t}^{*}$ such that $\left(\alpha_{i}, \alpha_{i}\right) \in \mathbf{Z}_{>0}$ and $\left\langle h_{i}, \lambda\right\rangle=2\left(\alpha_{i}, \lambda\right) /\left(\alpha_{i}, \alpha_{i}\right)$ for $\lambda \in t^{*}$. Let $\left\{\Lambda_{i}\right\}_{i \in I}$ be the dual base of $\left\{h_{i}\right\}$ and set $P=\sum \mathbf{Z} \Lambda_{i}$ and $P^{*}=\sum \mathbf{Z} h_{i}$. Then the q-analogue $U_{q}(\mathfrak{g})$ is the algebra over $\mathbf{Q}(q)$ generated by $e_{i}, f_{i}$ and $q^{h}\left(h \in P^{*}\right)$ satisfying the following relations:

$$
\begin{aligned}
& q^{h}=1 \text { if } h=0 \text { and } q^{h} q^{h^{\prime}}=q^{h+h^{\prime}}, \\
& q^{h} e_{j} q^{-h}=q^{\left\langle h, \alpha_{j}\right\rangle} e_{j} \text { and } q^{h} f_{j} q^{-h}=q^{-\left\langle h, \alpha_{j}\right\rangle} f_{j}, \\
& {\left[e_{i}, f_{j}\right]=\delta_{i, j} \frac{t_{i}-t_{i}^{-1}}{q_{i}-q_{i}^{-1}} \text {, where } q_{i}=q^{\left(\alpha_{i}, \alpha_{i}\right)} \text { and } t_{i}=q^{\left(\alpha_{i}, \alpha_{i}\right) h_{i}},} \\
& \quad \sum_{\mu=0}^{b} e_{i}^{(\mu)} e_{j} e_{i}^{(b-\mu)}=\sum_{\mu=0}^{b} f_{i}^{(\mu)} f_{j} f_{i}^{(b-\mu)}=0 .\left(i \neq j \text { and } b=1-\left\langle h_{i}, \alpha_{j}\right\rangle\right),
\end{aligned}
$$

where $e_{i}^{(k)}=e_{i}^{k} /[k]_{i} ! f_{i}^{(k)}=f_{i}^{k} /[k]_{i} !, \quad[n]_{i}=\left(q_{i}^{n}-q_{i}^{-n}\right) /\left(q_{i}-q_{i}^{-1}\right)$ and $[k]_{i} !=$ $\prod_{n=1}^{k}[n]_{i}$.

The comultiplication $\Delta: U_{q}(\mathfrak{g}) \rightarrow U_{q}(\mathfrak{g}) \otimes U_{q}(\mathrm{~g})$ is given by $\Delta\left(q^{h}\right)=q^{h} \otimes q^{h}$, $\Delta\left(e_{i}\right)=e_{i} \otimes t_{i}^{-1}+1 \otimes e_{i}$ and $\Delta\left(f_{i}\right)=f_{i} \otimes 1+t_{i} \otimes f_{i}$. If $M$ and $N$ are $U_{q}(\mathfrak{g})-$ modules, then by this comultiplication $M \otimes N$ is also $U_{q}(g)$-module.

For a finite-dimensional $U_{q}(\mathrm{~g})$-module $M$ and $\lambda \in P$, we set $M_{\lambda}=\{u \in M$; $\left.t_{i} u=q^{2\left(\alpha_{i}, \lambda\right)} u\right\}$. We call $M$ integrable if $M=\bigoplus M_{\lambda}$. Then we have

$$
M_{\lambda}=\underset{k \geqq 0,-\left\langle h_{i}, \lambda\right\rangle}{\bigoplus_{i}} f_{i}^{(k)}\left(M_{\lambda+k \alpha_{i}} \cap \operatorname{Ker} e_{i}\right) .
$$

We define the operators $\tilde{e}_{i}, \tilde{f}_{i}$ acting on $M$ by $\tilde{e}_{i} f_{i}^{(k)} u=f_{i}^{(k-1)} u$ and $\tilde{f}_{i} f_{i}^{(k)} u=f_{i}^{(k+1} u$, for $u \in M_{\lambda+k \alpha_{i}} \cap \operatorname{Ker} e_{i}$ and $(\lambda, k)$ as above.

Definition 1.1.1. Let $A$ be the ring of rational functions regular at $q=0$. A pair $(L, B)$ is called a crystal base of a finite-dimensional integrable representation $M$ if the following conditions are satisfied;

(1) $L$ is a free sub- $A$-module of $M$ such that $\mathbf{Q}(q) \otimes_{A} L \cong M$.

(2) $B$ is a base of the $\mathbf{Q}$-vector space $L / q L$.

(3) $L=\oplus L_{\lambda}, B=\coprod B_{\lambda}$, where $L_{\lambda}=L \cap M_{\lambda}$ and $B_{\lambda}=B \cap\left(L_{\lambda} / q L_{\lambda}\right)$.

(4) $\tilde{f}_{i} L \subset L$, and $\tilde{e}_{i} L \subset L$.

(5) $\tilde{f}_{i} B \subset B \cup\{0\}$ and $\tilde{e}_{i} B \subset B \cup\{0\}$.

(6) For $u, v \in B$ and $i \in I, u=\tilde{e}_{i} v$ if and only if $v=\tilde{f}_{i} u$.

We call $L$ crystal lattice and $B$ crystal.

Then the following results are proved in [K1] for $\mathrm{g}=A_{n}, B_{n}, C_{n}$ and $D_{n}$ and in [K2] in the general case. Let $\lambda \in P_{+}=\left\{\lambda \in t^{*} ;\left\langle h_{i}, \lambda\right\rangle \in \mathbf{Z}_{\geqq 0}\right\}$ and $V(\lambda)$ the 
irreducible integrable $U_{q}(\mathfrak{g})$-module generated by the highest weight vector $u_{\lambda}$ with weight $\lambda$. We set $L(\lambda)=\sum A \tilde{f}_{i_{1}} \ldots \tilde{f}_{i_{k}} u_{\lambda}$ and $B(\lambda)=\left\{\tilde{f}_{i_{1}} \ldots \tilde{f}_{i_{k}} u_{\lambda} \bmod q L(\lambda)\right\} \backslash\{0\}$ $\subset L(\lambda) / q L(\lambda)$, where $i_{j}$ runs over $I$.

Theorem 1.1.2. $(L(\lambda), B(\lambda))$ is a crystal base of $V(\lambda)$.

Theorem 1.1.3. If $(L, B)$ is a crystal base of an integrable $U_{q}(\mathfrak{g})$-module $M$, then there is an isomorphism: $M \cong \bigoplus_{j} V\left(\lambda_{j}\right)$ by which $(L, B) \cong \bigoplus_{j}\left(L\left(\lambda_{j}\right), B\left(\lambda_{j}\right)\right)$.

Corollary 1.1.4. Let $M$ and $(L, B)$ be as above. Then we obtain

$$
M \cong \bigoplus_{b \in B^{h}} V(w t(b)), \quad \text { where } B^{h}=\left\{b \in B ; \tilde{e}_{i} b=0 \text { for any } i\right\} .
$$

We call an element of $B^{h}$ a highest weight element of $B$.

Theorem 1.1.5. Let $\left(L_{j}, B_{j}\right)$ be a crystal base of an integrable $U_{q}(\mathrm{~g})$-module $M_{j}$ $(j=1,2)$. Set $L=L_{1} \otimes{ }_{A} L_{2} \subset M_{1} \otimes M_{2}$ and $B=\left\{b_{1} \otimes b_{2} ; b_{j} \in B_{j}(j=1,2)\right\}$ $\subset L / q L$. Then we have

$$
(L, B) \text { is a crystal base of } M_{1} \otimes M_{2} .
$$

$$
\begin{aligned}
& \tilde{f}_{i}\left(b_{1} \otimes b_{2}\right)= \begin{cases}\tilde{f}_{i} b_{1} \otimes b_{2} & \text { if } \varphi_{i}\left(b_{1}\right)>\varepsilon_{i}\left(b_{2}\right), \\
b_{1} \otimes \tilde{f}_{i} b_{2} & \text { if } \varphi_{i}\left(b_{1}\right) \leqq \varepsilon_{i}\left(b_{2}\right) .\end{cases} \\
& \tilde{e}_{i}\left(b_{1} \otimes b_{2}\right)= \begin{cases}b_{1} \otimes \tilde{e}_{i} b_{2} & \text { if } \varphi_{i}\left(b_{1}\right)<\varepsilon_{i}\left(b_{2}\right), \\
\tilde{e}_{i} b_{1} \otimes b_{2} & \text { if } \varphi_{i}\left(b_{1}\right) \geqq \varepsilon_{i}\left(b_{2}\right) .\end{cases}
\end{aligned}
$$

Here, $\varepsilon_{i}(b)=\max \left\{k \geqq 0 ; \tilde{e}_{i}^{k} b \neq 0\right\}$ and $\varphi_{i}(b)=\max \left\{k \geqq 0 ; \tilde{f}_{i}^{k} b \neq 0\right\}$.

Corollary 1.1.6. For $b_{j} \in B_{j}(j=1,2)$, we have

$$
\begin{aligned}
\varepsilon_{i}\left(b_{1} \otimes b_{2}\right) & =\max \left(\varepsilon_{i}\left(b_{1}\right), \varepsilon_{i}\left(b_{1}\right)+\varepsilon_{i}\left(b_{2}\right)-\varphi_{i}\left(b_{1}\right)\right), \\
\varphi_{i}\left(b_{1} \otimes b_{2}\right) & =\max \left(\varphi_{i}\left(b_{2}\right), \varphi_{i}\left(b_{1}\right)+\varphi_{i}\left(b_{2}\right)-\varepsilon_{i}\left(b_{2}\right)\right) .
\end{aligned}
$$

Definition 1.1.7. A crystal graph of a crystal base $(L, B)$ is the colored and oriented graph $B$, with the arrows: $u \stackrel{i}{\rightarrow} v$ if and only if $v=\tilde{f}_{i} u$.

Let $M_{i}(i=1,2)$ and $B$ be as in Theorem 1.1.5, and $B^{h}$ be the set of highest weight elements of $B$. By Corollary 1.1.4 and Theorem 1.1.5, we get

$$
M_{1} \otimes M_{2} \cong \bigoplus_{b_{1} \otimes b_{2} \in B^{h}} V\left(w t\left(b_{1} \otimes b_{2}\right)\right) .
$$

The aim of this paper is to give the explicit procedure for obtaining such $b_{1}$ and $b_{2}$.

\section{Review of Crystal Graphs}

In this section, we shall summarize the results of [K-N], which gives an explicit description of crystal graphs of $U_{q}(\mathfrak{g})$-modules $\left(\mathfrak{g}=A_{n}, B_{n}, C_{n}, D_{n}\right)$. We omit the rule of arrows.

2.1. Crystals for $U_{q}\left(A_{n}\right)$-modules. We shall treat the $A_{n}$-case. We set $\varepsilon_{1}=\Lambda_{1}$, $\varepsilon_{i}=\Lambda_{i+1}-\Lambda_{i}$ for $2 \leqq i \leqq n$ and $\varepsilon_{n+1}=-\left(\varepsilon_{1}+\cdots+\varepsilon_{n}\right)$. Define $\alpha_{i}=\varepsilon_{i}-\varepsilon_{i+1}$. 
The crystal graph $B\left(V_{\square}\right)$ of the vector representation $V_{\square}$ is easily obtained by explicit construction. It is labelled by $\{[i] ; i=1, \ldots, n+1\}$ and the crystal graph is

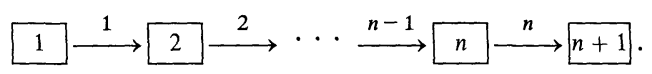

Here, $i$ has weight $\varepsilon_{i}$. Graph (2.1.1) implies

$$
\tilde{e}_{i} j=\delta_{i, j-1} j-1, \tilde{f}_{i} j=\delta_{i, j} j+1 .
$$

Let $V\left(\Lambda_{N}\right)$ be the irreducible representation with the fundamental weight $\Lambda_{N}$ $(1 \leqq N \leqq n)$ as a highest weight. We embed $V\left(\Lambda_{N}\right)$ into $V_{\square}^{\otimes N}$. Accordingly, $B\left(\Lambda_{N}\right)$ is embedded into $B\left(V_{\square}\right)^{\otimes N}$. We have that $B\left(\Lambda_{N}\right)$ consists of $i_{1} \otimes \cdots \otimes i_{N}$ with $1 \leqq i_{1}<\cdots<i_{N} \leqq n+1$. The base $\left.u_{\Lambda_{N}}=1\right] \otimes 2 \otimes \cdots \otimes[N$ is annihilated by all $\tilde{e}_{i}$ and it has weight $\Lambda_{N}=\varepsilon_{1}+\cdots+\varepsilon_{N}$, then the crystal graph $B\left(\Lambda_{N}\right)$ is the connected component of $B\left(V_{\square}\right)^{\otimes N}$ containing $1 \otimes \cdots \otimes N$. We write

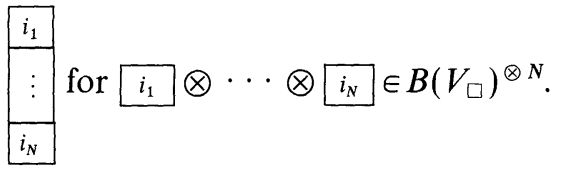

Let $\lambda=\sum_{i=1}^{p} \Lambda_{l_{2}}\left(1 \leqq l_{1} \leqq l_{2} \leqq \cdots \leqq l_{p} \leqq n\right)$ be a dominant integral weight.

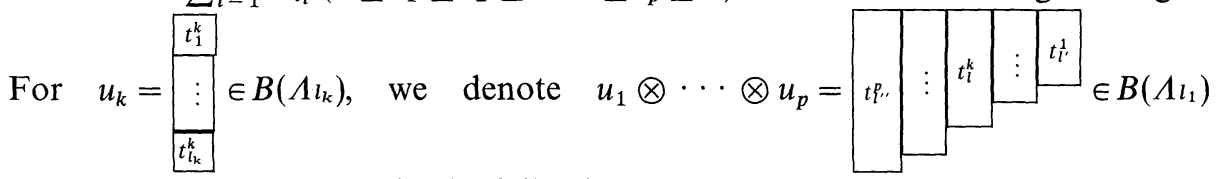
$\otimes \cdots \otimes B\left(\Lambda l_{p}\right)$. We obtain the following:

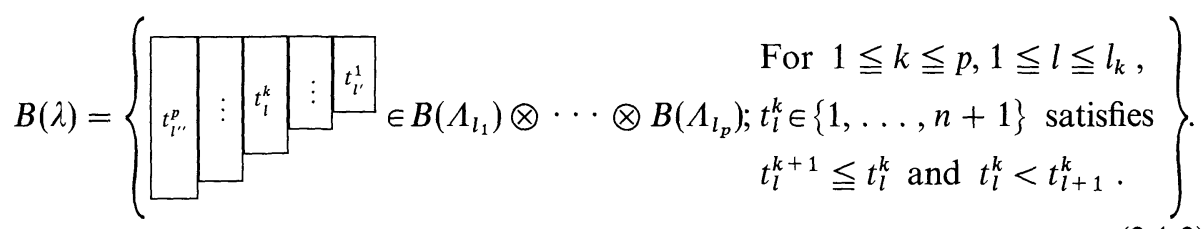

An element of $B(\lambda)$ is called a semi-standard A-tableau of shape $\lambda$. Note that semi-standard A-tableaux coincide with usual semi-standard tableaux, so in the case $\mathrm{g}=\mathrm{gl}(n+1), B(\lambda)$ is also given by the same rule as the $A_{n}$-case.

2.2. Crystals for $U_{q}\left(C_{n}\right)$-modules. Let $\left(\varepsilon_{1}, \ldots, \varepsilon_{n}\right)$ be the orthonormal base of the dual of the Cartan subalgebra of $C_{n}$ such that $\alpha_{i}=\varepsilon_{i}-\varepsilon_{i+1}(1 \leqq i<n)$ and $\alpha_{n}=2 \varepsilon_{n}$ are the simple roots. Hence, $\alpha_{n}$ is the long root and $\alpha_{1}, \ldots, \alpha_{n-1}$ are short roots. Let $\left\{\Lambda_{i}\right\}_{1 \leqq i \leqq n}$ be the dual base of $\left\{h_{i}\right\}_{1 \leqq i \leqq n}$. Hence $\Lambda_{i}=\varepsilon_{1}+\cdots+\varepsilon_{i}(1 \leqq i \leqq n)$.

The crystal graph $B\left(V_{\square}\right)$ of the vector representation $V_{\square}$ is described as follows. It is labelled by $\{i, \bar{i}, 1 \leqq i \leqq n\}$, where $i$ has weight $\varepsilon_{i}$ and has $\bar{i}$ has weight $-\varepsilon_{i}$. Its crystal graph is

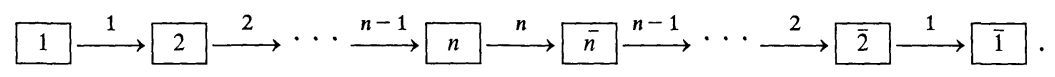


Similarly to the $A_{n}$-case, the connected component of the crystal graph $B\left(V_{\square}\right)^{\otimes N}$ containing $u_{\Lambda_{N}}=\square \otimes \square \otimes \cdots \otimes N$ is isomorphic to $B\left(\Lambda_{N}\right)$.

We give the linear order on $\{i, \bar{i} ; 1 \leqq i \leqq n\}$ by $1 \prec 2 \prec \cdots n \prec \bar{n} \prec \cdots$ $\prec \overline{2} \prec \overline{1}$. By using the same notation as in 2.1 , we have

$$
B\left(\Lambda_{N}\right)=\left\{\begin{array}{ll}
\text { (1) } 1 \leqq i_{1} \prec \cdots \prec i_{N} \leqq \overline{1} \\
\hline i_{1} \\
\vdots \vdots \\
\hdashline i_{N}
\end{array} \in B\left(V_{\square}\right)^{\otimes N} ; \text { (2) } \begin{array}{rl}
\text { if } i_{k}=p \text { and } i_{l}=\bar{p} \\
\text { then } k+(N-l+1) \leqq p
\end{array}\right\} .
$$

Next, we shall give the crystal of $V\left(\Lambda_{M}+\Lambda_{N}\right)$ with $1 \leqq M \leqq N \leqq n$. By embedding $V\left(\Lambda_{M}+\Lambda_{N}\right)$ into $V\left(\Lambda_{M}\right) \otimes V\left(\Lambda_{N}\right), B\left(\Lambda_{M}+\Lambda_{N}\right)$ is the connected component of $B\left(\Lambda_{M}\right) \otimes B\left(\Lambda_{N}\right)$ containing $u_{\Lambda_{M}} \otimes u_{\Lambda_{N}}$.

For $u=$\begin{tabular}{|c|}
\hline$j_{1}$ \\
\hline$\vdots$ \\
\hline$\vdots$ \\
\hline$j_{M}$
\end{tabular}$\in B\left(\Lambda_{M}\right)$ and $v=$\begin{tabular}{|c|c|c|}
\hline$i_{1}$ \\
\hline$\vdots$ \\
\hline$i_{N}$ \\
\hline
\end{tabular}$\in B\left(\Lambda_{N}\right), u \otimes v$ will be denoted by \begin{tabular}{|c|c|}
\hline$i_{1}$ & $j_{1}$ \\
\hline$\vdots$ & $\vdots$ \\
\hline$i_{N}$ & $j_{M}$ \\
\hline
\end{tabular}

Definition 2.2.1. For $1 \leqq a \leqq b \leqq n$ and $u$, $v$ as above, we say that $u \otimes v \in B\left(\Lambda_{M}\right) \otimes$ $B\left(\Lambda_{N}\right)$ is in the $(a, b)$-configuration if $u \otimes v$ satisfies the following;

there exist $1 \leqq p \leqq q<r \leqq s \leqq M$ such that $i_{p}=a, j_{q}=b, j_{r}=\bar{b}, j_{s}=\bar{a}$ or $i_{p}=a, i_{q}=b, i_{r}=\bar{b}, j_{s}=\bar{a}$.

This definition includes the case $a=b, p=q$ and $r=s$. Now, we define $p(a, b ; u \otimes v)=(q-p)+(s-r)$. We obtain the following;

$$
\begin{aligned}
& B\left(\Lambda_{M}+\Lambda_{N}\right)=
\end{aligned}
$$

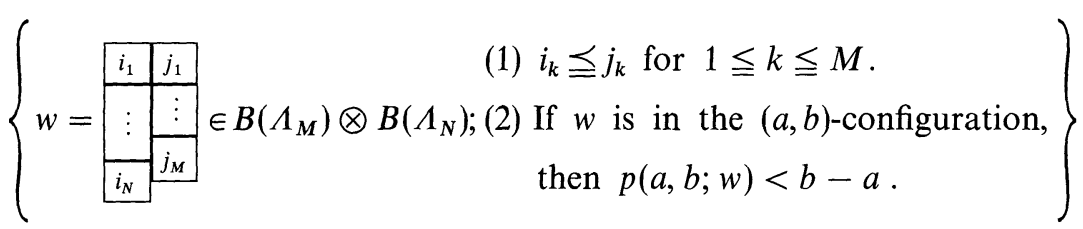

Let $\lambda=\sum_{i=1}^{p} \Lambda_{l_{1}}\left(1 \leqq l_{1} \leqq \cdots \leqq l_{p} \leqq n\right)$ be a dominant integral weight. Using the same notation as in (2.1.3), we obtain;

$$
B(\lambda)=\left\{\left[\begin{array}{c|c|c|c|c|}
t_{l^{\prime}} & \vdots & \vdots & t_{l}^{1}
\end{array} \in B\left(\Lambda_{l_{1}}\right) \otimes \cdots \otimes B\left(\Lambda_{l_{p}}\right) ; \begin{array}{l}
u_{k} \otimes u_{k+1} \in B\left(\Lambda_{l_{k}}+\Lambda_{k+1}\right) \\
\text { for any } k .
\end{array}\right\} .\right.
$$

An element of $B(\lambda)$ is called a semi-standard $C$-tableau of shape $\lambda$.

2.3. Crystals for $U_{q}\left(B_{n}\right)$-modules. Let $\left(\varepsilon_{1}, \varepsilon_{2}, \ldots, \varepsilon_{n}\right)$ be the orthonormal base of the dual of the Cartan subalgebra of $B_{n}$ such that $\alpha_{i}=\varepsilon_{i}-\varepsilon_{i+1}(1 \leqq i<n)$ and 
$\alpha_{n}=\varepsilon_{n}$ are simple roots. Hence, $\alpha_{1}, \ldots, \alpha_{n-1}$ are long roots and $\alpha_{n}$ is the short root. Let $\left\{\Lambda_{i}\right\}_{1 \leqq i \leqq n}$ be the dual base of $\left\{h_{i}\right\}_{1 \leqq i \leqq n}$. Hence $\Lambda_{i}=\varepsilon_{1}+\cdots+\varepsilon_{i}$ $(i=1, \ldots, n-1)$ and $\Lambda_{n}=\left(\varepsilon_{1}+\cdots+\varepsilon_{n}\right) / 2$.

The crystal graph $B\left(V_{\square}\right)$ of the vector representation $V_{\square}$ is described as follows. It is labelled by $\left\{[i, \bar{i}, 1 \leqq i \leqq n\} \cup\left\{\left[0\right.\right.\right.$, , where $i$, has weight $\varepsilon_{i}, \bar{i}$ has weight $-\varepsilon_{i}$ and 0 has weight 0 . The crystal graph of $V_{\square}$ is

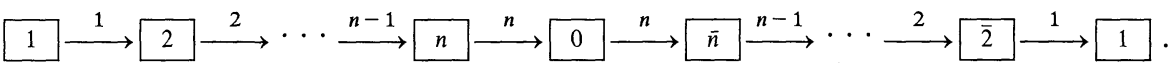

Next, we set $\omega_{i}=\varepsilon_{1}+\cdots+\varepsilon_{i}(1 \leqq i \leqq n)$. Note that $\omega_{i}=\Lambda_{i}(1 \leqq i<n)$ and $\omega_{n}=2 \Lambda_{n}$. The representation $V\left(\omega_{N}\right)$ can be embedded into $V_{\square}^{\otimes N}(1 \leqq N \leqq n)$.

We give the linear order on $\{i, \bar{i} ; 1 \leqq i \leqq n\} \cup\{0\}$ by

$$
1<2<\cdots<n<0<\bar{n} \prec \cdots \prec \overline{2}<\overline{1} \text {. }
$$

Then we obtain

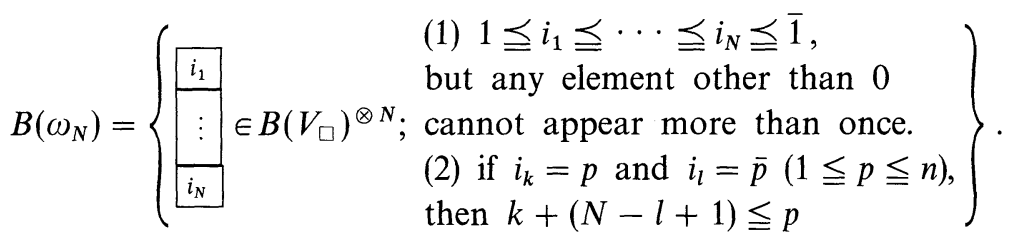

Next, we introduce the spin representation which is denoted $V_{\mathrm{sp}}$. This is the finite-dimensional irreducible representation with highest weight $\Lambda_{n}$. This is described explicitly by use of the following half-size tableaux. With the linear order on $\{1,2, \ldots, n, \bar{n}, \ldots, \overline{2}, \overline{1}\}$ as before, we set

$$
B_{\mathrm{sp}}=\left\{\begin{array}{ll}
i_{1} & \text { (1) } i_{j} \in\{1,2, \ldots, n, \bar{n}, \ldots, \overline{2}, \overline{1}\}, \\
\vdots \vdots ; & \text { (2) } i_{1} \prec \cdots \prec i_{n}, \\
\hline i_{n} & \text { (3) } i \text { and } \bar{i} \text { do not appear simultaneously }
\end{array}\right\},
$$

$V_{\mathrm{sp}}=\bigoplus_{v \in B_{\mathrm{sp}}} \mathbf{Q}(q) v$ and $L_{\mathrm{sp}}=\bigoplus_{v \in \boldsymbol{B}_{\mathrm{sp}}} A v$. If we define the actions of generators as in [K-N], $V_{\mathrm{sp}} \cong V\left(\Lambda_{n}\right)$ and $\left(L_{\mathrm{sp}}, B_{\mathrm{sp}}\right)$ is a crystal base of $V_{\mathrm{sp}}$.

Next, we shall give the crystal of $V\left(\omega_{M}+\omega_{N}\right)(1 \leqq M \leqq N \leqq n) . V\left(\omega_{M}+\omega_{N}\right)$ can be embedded into $V\left(\omega_{M}\right) \otimes V\left(\omega_{N}\right)$ uniquely.

\section{Definition 2.3.1.}

(1) For $1 \leqq a \leqq b<n$, we say that $w=u \otimes v \in B\left(\omega_{M}\right) \otimes B\left(\omega_{N}\right)$ is in the $(a, b)$ configuration if $w=u \otimes v$ satisfies the same condition as Definition 2.2.1.

(2) For $1 \leqq a<n$, we say that $w=u \otimes v \in B\left(\omega_{M}\right) \otimes B\left(\omega_{N}\right)$ is in the $(a, n)$-configuration if $w=u \otimes v$ holds the following;

there exist $1 \leqq p \leqq q<r=q+1 \leqq s \leqq M$ such that $i_{p}=a, j_{s}=\bar{a}$ and one of the following conditions is satisfied:

(i) $i_{q}$ and $i_{r}\left(=i_{q+1}\right)$ are $n, 0$ or $\bar{n}$.

(ii) $j_{q}$ and $j_{r}\left(=j_{q+1}\right)$ are $n$, 0 or $\bar{n}$. 
(3) We say that $w=u \otimes v \in B\left(\omega_{M}\right) \otimes B\left(\omega_{N}\right)$ is in the $(n, n)$-configuration if there exist $1 \leqq p<q \leqq M$ such that $i_{p}=n$ or 0 and $j_{q}=0$ or $\bar{n}$.

This definition includes the case $a=b, p=q$ and $r=s$.

Now, for $w$ in the $(a, b)$-configuration, we define $p(a, b ; w)=(q-p)+(s-r)$. (If $a=b=n$, we set $p(a, b ; w)=0$.)

We obtain

$$
\begin{aligned}
& B\left(\omega_{M}+\omega_{N}\right)= \\
& \left\{w=u \otimes v=\begin{array}{c|c|c|}
\hline i_{1} & j_{1} \\
\hline \vdots & \vdots \\
\hline i_{N} & j_{M}
\end{array} \in B\left(\omega_{M}\right) \otimes B\left(\omega_{N}\right) ; \quad \begin{array}{l}
w \text { satisfies following } \\
\text { (M.N.1) and (M.N.2) }
\end{array}\right\} .
\end{aligned}
$$

(M.N.1) $\quad i_{k} \leqq j_{k}$ for $1 \leqq k \leqq M$, but if $i_{k}=0$ or $j_{k}=0$, then $i_{k} \prec j_{k}$.

(M.N.2) If $w$ is in the $(a, b)$-configuration, then $p(a, b ; w)<b-a$.

We shall consider the crystal of $V\left(\omega_{M}+\Lambda_{n}\right)(1 \leqq M \leqq n)$.

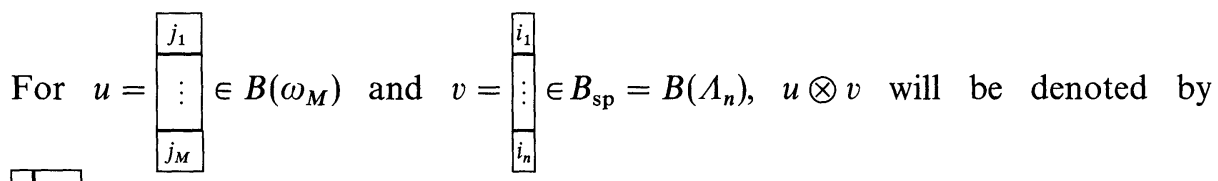

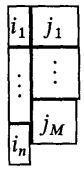

Definition 2.3.2. When $u \in B\left(\omega_{M}\right)$ and $v \in B_{\mathrm{sp}}$ have the above expression, for $1 \leqq a \leqq b \leqq n$ we say that $w=u \otimes v$ is in the $(a, b)$-configuration if $w$ satisfies the same condition as Definition 2.3.1. We define $p(a, b ; w)=(q-p)+(s-r)$. We have

$$
\begin{aligned}
& B\left(\omega_{M}+\Lambda_{n}\right)=
\end{aligned}
$$

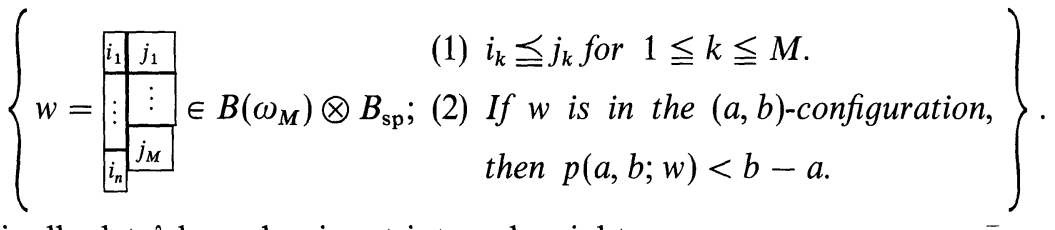

Finally, let $\lambda$ be a dominant integral weight.

Theorem 2.3.3. (i) Suppose $\left\langle h_{n}, \lambda\right\rangle$ is even. We can write $\lambda=\sum_{k=1}^{p} \omega_{l_{k}}$ with $l_{1} \leqq \cdots \leqq l_{p}$. We use the same notation as in (2.1.3). Then

$B(\lambda)=\left\{t_{t}^{p} \vdots \vdots t_{l^{\prime}}^{t^{\prime}} \vdots \vdots t_{l^{\prime \prime}}^{1} \in B\left(\omega_{l_{1}}\right) \otimes \cdots \otimes B\left(\omega_{l_{p}}\right) ; \begin{array}{c}u_{k} \otimes u_{k+1} \in B\left(\omega_{l_{k}}+\omega_{l_{k+1}}\right) \\ \text { for } k=1, \ldots, p-1\end{array}\right\}$. 
(ii) Suppose $\left\langle h_{n}, \lambda\right\rangle$ is odd. We can write $\lambda=\sum_{k=1}^{p-1} \omega_{l_{k}}+\Lambda_{n}$ with $l_{1} \leqq \cdots \leqq l_{p-1}$. For $u_{k} \in B\left(\omega_{l_{k}}\right)(1 \leqq k<p)$ (the same expression as above) and $u_{p}=\stackrel{\underbrace{t_{1}}_{1}}{\vdots} \in B_{\mathrm{sp}}$, denote $u_{1} \otimes \cdots \otimes u_{p}=t_{t^{p}}: t_{t^{\prime}}^{k^{\prime}}:{ }^{t_{l^{\prime \prime}}^{\prime \prime}} \in B\left(\omega_{l_{1}}\right) \otimes \cdots \otimes B\left(\omega_{l_{p-1}}\right) \otimes B_{\mathrm{sp}}$, then we obtain

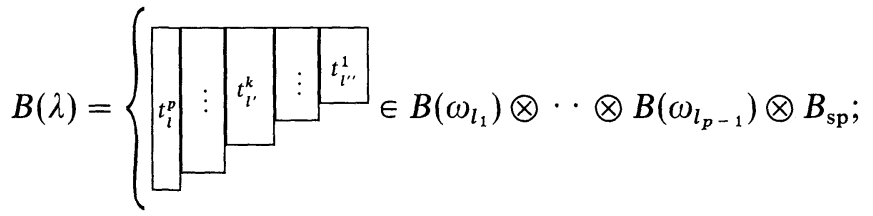

$$
\begin{aligned}
& \left.\begin{array}{l}
\text { For } k=1, \ldots, p-1 \\
u_{k} \otimes u_{k+1} \in B\left(\omega_{l_{k}}+\omega_{l_{k+1}}\right) \\
u_{p-1} \otimes u_{p} \in B\left(\omega_{l_{p-1}}+\Lambda_{n}\right)
\end{array}\right\}
\end{aligned}
$$

An element of $B(\lambda)$ is called a semi-standard B-tableau of shape $\lambda$.

2.4. Crystals for $U_{q}\left(D_{n}\right)$-modules. Let $\left(\varepsilon_{1}, \varepsilon_{2}, \ldots, \varepsilon_{n}\right)$ be the orthonormal base of the dual of the Cartan subalgebra of $D_{n}$ such that $\alpha_{i}=\varepsilon_{i}-\varepsilon_{i+1}(1 \leqq i<n)$ and $\alpha_{n}=\varepsilon_{n-1}+\varepsilon_{n}$ form the set of simple roots. Let $\left\{\Lambda_{i}\right\}_{1 \leqq i \leqq n}$ be the dual base of $\left\{h_{i}\right\}_{1 \leqq i \leqq n}$. Hence $\Lambda_{i}=\varepsilon_{1}+\cdots+\varepsilon_{i}(i=1, \ldots, n-2)$ and $\Lambda_{n-1}=\left(\varepsilon_{1}+\cdots\right.$ $\left.+\varepsilon_{n-1}-\varepsilon_{n}\right) / 2 . \Lambda_{n}=\left(\varepsilon_{1}+\cdots+\varepsilon_{n-1}+\varepsilon_{n}\right) / 2$.

The crystal graph $B\left(V_{\square}\right)$ of the vector representation $V_{\square}$ is the following. It is labelled by $\{i, \bar{i}, \bar{i} ; 1 \leqq i \leqq n\}$, where $i$ has weight $\varepsilon_{i}$ and $\bar{i}$ has weight $-\varepsilon_{i}$. The crystal graph of $V_{\square}$ is

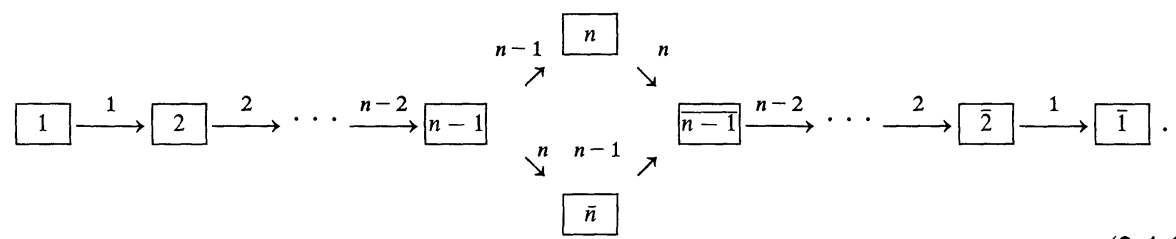

Next, we set $\omega_{i}=\varepsilon_{1}+\cdots+\varepsilon_{i}(1 \leqq i \leqq n)$ and $\omega_{n+1}=\varepsilon_{1}+\cdots+\varepsilon_{n-1}-\varepsilon_{n}$. Note that $\omega_{i}=\Lambda_{i} \quad(1 \leqq i \leqq n-2), \quad \omega_{n-1}=\Lambda_{n-1}+\Lambda_{n}, \quad \omega_{n}=2 \Lambda_{n} \quad$ and $\omega_{n+1}=2 \Lambda_{n-1}$.

The representation $V\left(\omega_{N}\right)(1 \leqq N \leqq n)$ (respectively $\left.V\left(\omega_{n+1}\right)\right)$ is embedded into $V_{\square}^{\otimes N}$ (respectively $V_{\square}^{\otimes n}$ ). We give the ordering on $\{i, \bar{i} ; 1 \leqq i \leqq n\}$ by;

$$
1 \prec 2 \prec \cdots n-1 \prec_{\bar{n}}^{n} \prec \overline{n-1} \prec \cdots \prec \overline{2} \prec \overline{1} .
$$


Note that there is no order between $n$ and $\bar{n}$. Using the same expression as before, we obtain for $1 \leqq N \leqq n$,

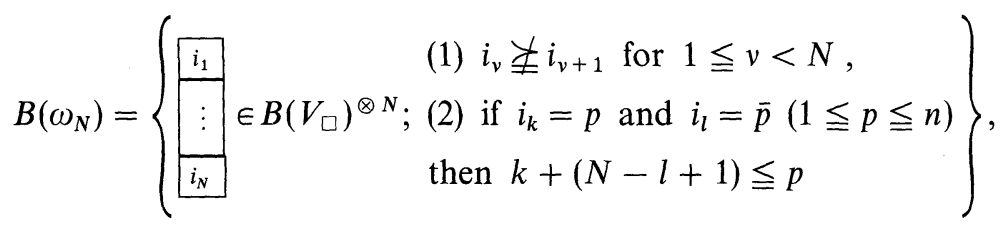

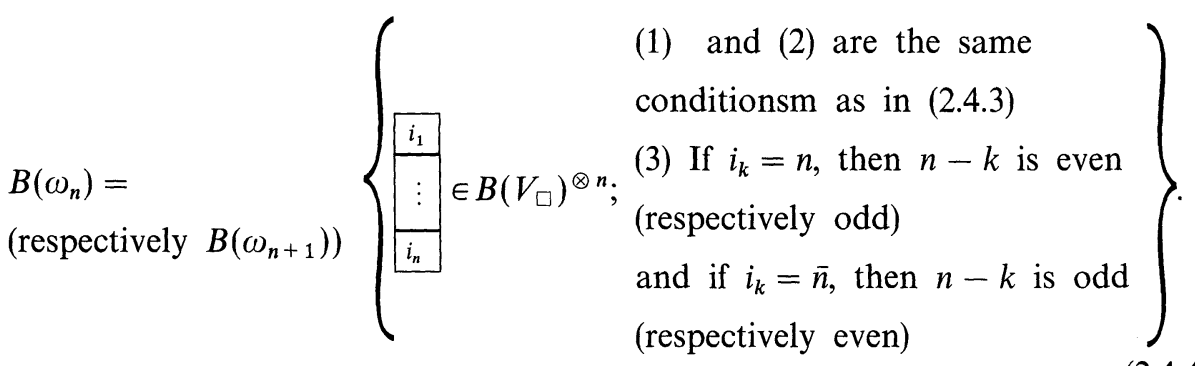

Remark 2.4.1. The condition (1) in (2.4.3) is equivalent to saying that for any $v$ either $i_{v} \prec i_{v+1}$, or $\left(i_{v}, i_{v+1}\right)=(n, \bar{n})$ or $(\bar{n}, n)$.

For the algebra $U_{q}\left(D_{n}\right)$, there are two spin representations $V_{\mathrm{sp}}^{(+)}$and $V_{\mathrm{sp}}^{(-)}$. They are the finite-dimensional irreducible representations with highest weight $\Lambda_{n}$ and $\Lambda_{n-1}$ respectively. They have the explicit description as follows. First we give the order on $P=\{1,2, \ldots, n, \bar{n}, \ldots, \overline{2}, \overline{1}\}$ as (2.4.2). Next, we use the half-size tableaux and set

$B_{\mathrm{sp}}^{(+)}\left(\right.$respectively $\left.B_{\mathrm{sp}}^{(-)}\right)=$

$$
\left\{v=\begin{array}{ll}
i_{1} & \begin{array}{l}
\text { (1) } i_{j} \in P \\
\text { (2) } i_{1} \prec i_{2} \prec \cdots \prec i_{n-1} \prec i_{n}
\end{array} \\
\hdashline \vdots ; & \text { (3) } i \text { and } \bar{i} \text { cannot appear simultaneously, } \\
\text { (4) If } i_{k}=n, \text { then } n-k \text { is even (respectively odd) } \\
\text { (5) If } i_{k}=\bar{n} \text {, then } n-k \text { is odd (respectively even) }
\end{array}\right\},
$$

$V_{\mathrm{sp}}^{( \pm)}=\bigoplus_{v \in B_{\mathrm{sp}}^{( \pm)}} \mathbf{Q}(q) v$ and $L_{\mathrm{sp}}^{( \pm)}=\bigoplus_{v \in B_{\mathrm{sp}}^{( \pm)}} A v$. If we give the actions of generators as in $[\mathrm{K}-\mathrm{N}]$, then $V_{\mathrm{sp}}^{(+)} \cong V\left(\Lambda_{n}\right), V_{\mathrm{sp}}^{(-)} \cong V\left(\Lambda_{n-1}\right)$ and $\left(L_{\mathrm{sp}}^{( \pm)}, B_{\mathrm{sp}}^{( \pm)}\right)$is the crystal base of $V_{\mathrm{sp}}^{( \pm)}$.

Next, we shall describe the crystal of $V\left(\omega_{M}+\omega_{N}\right)(1 \leqq M \leqq N \leqq n+1)$. For $u \otimes v \in B\left(\omega_{M}\right) \otimes B\left(\omega_{N}\right)$ we use the same expression as before.

Definition 2.4.2. Let $1 \leqq M \leqq N \leqq n+1$ such that $(M, N) \neq(n, n+1)$.

(1) For $1 \leqq a \leqq b<n$, we say that $u \otimes v \in B\left(\omega_{M}\right) \otimes B\left(\omega_{N}\right)$ is in the $(a, b)$-configuration if $u \otimes v$ satisfies the same condition as Definition 2.2.1.

(2) For $1 \leqq a<n$, we say that $u \otimes v \in B\left(\omega_{M}\right) \otimes B\left(\omega_{N}\right)$ is in the $(a, n)$-configuration if $u \otimes v$ satisfies the following; 
there exist $1 \leqq p \leqq q<r=q+1 \leqq s \leqq M$ such that $\left(i_{p}, j_{s}\right)=(a, \bar{a})$ or $(\bar{a}, a)$ and one of the following conditions is satisfied:

(i) $i_{q}$ and $i_{r}\left(=i_{q+1}\right)$ are $n$ or $\bar{n}$,

(ii) $j_{q}$ and $j_{r}\left(=j_{q+1}\right)$ are $n$ or $\bar{n}$.

If $w$ is in the $(a, b)$-configuration, we define $p(a, b ; w)=(q-p)+(s-r)$.

(3) For $1 \leqq a<n$, we say that $u \otimes v \in B\left(\omega_{M}\right) \otimes B\left(\omega_{N}\right)$ is in the a-odd-configuration if $u \otimes v$ satisfies the following; there exist $1 \leqq p \leqq q<r \leqq s \leqq M$ such that (a) $r-q+1$ is odd, (b) $i_{p}=a$ and $j_{s}=\bar{a}$, (c) $j_{q}=n$ and $i_{r}=\bar{n}$, or $j_{q}=\bar{n}$ and $i_{r}=n$.

(4) For $1 \leqq a<n$, we say that $u \otimes v \in B\left(\omega_{M}\right) \otimes B\left(\omega_{N}\right)$ is in the a-even-configuration if $u \otimes v$ satisfies the following; there exist $1 \leqq p \leqq q<r \leqq s \leqq M$ such that $(a)$ $r-q+1$ is even, $(b) i_{p}=a$ and $j_{s}=\bar{a},(c) j_{q}=n$ and $i_{r}=n$, or $j_{q}=\bar{n}$ and $i_{r}=\bar{n}$.

If $w \in B\left(\omega_{M}\right) \otimes B\left(\omega_{N}\right)$ is in the $a$-odd or even-configuration, we define $q(a ; w)=s-p$. Then we have, for $1 \leqq M \leqq N \leqq n+1$, with $(M, N) \neq(n, n+1)$.

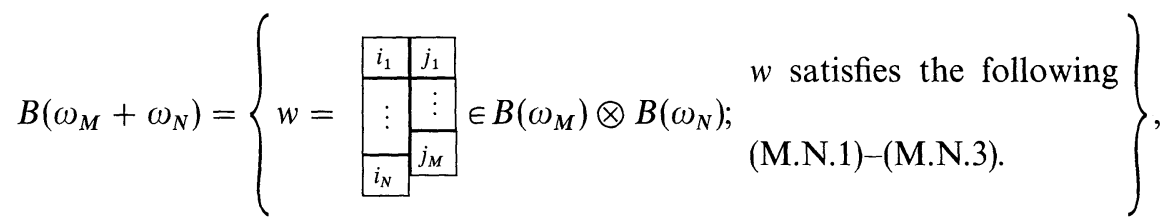

(M.N.1) $i_{k} \leqq j_{k}$ for $1 \leqq k \leqq M$.

(M.N.2) If $w$ is in the $(a, b)$-configuration, then $p(a, b ; w)<b-a$.

(M.N.3) If $w$ is in the a-odd-configuration or the $a$-even-configuration, then $q(a ; w)<n-a$.

Next, we shall treat the representations $V\left(\omega_{M}+\Lambda_{n}\right)(1 \leqq M \leqq n)$ and $V\left(\omega_{N}+\Lambda_{n-1}\right)(1 \leqq N \leqq n+1, N \neq n)$. They can be embedded into respectively $V\left(\omega_{M}\right) \otimes V_{\mathrm{sp}}^{(+)}$and $V\left(\omega_{N}\right) \otimes V_{\mathrm{sp}}^{(-)}$with multiplicity free. For $u \otimes v \in B\left(\omega_{M}\right) \otimes B_{\mathrm{sp}}^{(+)}$ and $B\left(\omega_{N}\right) \otimes B_{\mathrm{sp}}^{(-)}$, we use the same expression as in 2.3 .

\section{Definition 2.4.3.}

(1) $u \otimes v \in B\left(\omega_{M}\right) \otimes B_{\mathrm{sp}}^{(+)}(1 \leqq M \leqq n)$ or $B\left(\omega_{N}\right) \otimes B_{\mathrm{sp}}^{(-)}(1 \leqq N \leqq n+1, N \neq n)$ is in the $(a, b)$-configuration $(1 \leqq a \leqq b \leqq n)$ if $u \otimes v$ satisfies the same condition as Definition 2.4.2. (1), (2).

(2) $u \otimes v \in B\left(\omega_{M}\right) \otimes B_{\mathrm{sp}}^{(+)}(1 \leqq M \leqq n)$ or $B\left(\omega_{N}\right) \otimes B_{\mathrm{sp}}^{(-)}(1 \leqq N<n)$ is in the a-odd (respectively even)-configuration $(1 \leqq a \leqq n)$ if $u \otimes v$ satisfies the same condition as Definition 2.4.2. (3) (respectively (4)).

$p(a, b ; w)$ and $q(a ; w)$ are the ones defined in Definition 2.4.2. Then we obtain,

$B\left(\omega_{M}+\Lambda_{n}\right)=$

(respectively $\left.B\left(\omega_{M}+\Lambda_{n-1}\right)\right)$

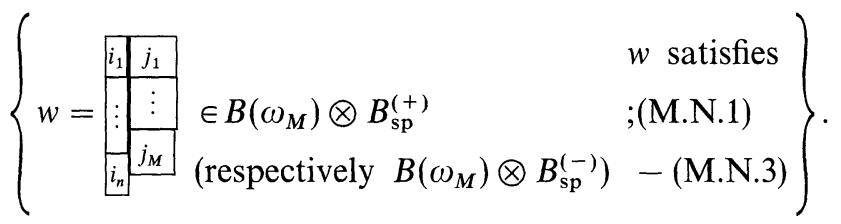


Let $\lambda=\sum_{i=1}^{n} m_{i} \Lambda_{i}\left(m_{i} \in \mathbf{Z}_{\geqq 0}\right)$ be a dominant integral weight of $D_{n}$. Now, we shall rewrite $\lambda$ by use of $\omega_{M}, \Lambda_{n}$ and $\Lambda_{n-1}$. By the definition of $\omega_{M}$, we have $\Lambda_{i}=\omega_{i}$ $(1 \leqq i \leqq n-2), \Lambda_{n-1}+\Lambda_{n}=\omega_{n-1}, 2 \Lambda_{n}=\omega_{n}$ and $2 \Lambda_{n-1}=\omega_{n+1}$. Hence, any $\lambda \in P_{+}$can be written in one of the following forms;

$$
\begin{aligned}
& \text { (W1) } \lambda=\sum_{i=1}^{n} m_{i} \omega_{i}\left(m_{i} \in \mathbf{Z}_{\geqq 0}\right) . \\
& \text { (W2) } \lambda=\sum_{i=1}^{n} m_{i} \omega_{i}+\Lambda_{n}\left(m_{i} \in \mathbf{Z}_{\geqq 0}\right) . \\
& \text { (W3) } \lambda=\sum_{i=1}^{n-1} m_{i} \omega_{i}+m_{n+1} \omega_{n+1}\left(m_{i} \in \mathbf{Z}_{\geqq 0}\right) . \\
& \text { (W4) } \lambda=\sum_{i=1}^{n-1} m_{i} \omega_{i}+m_{n+1} \omega_{n+1}+\Lambda_{n-1}\left(m_{i} \in \mathbf{Z}_{\geqq 0}\right) .
\end{aligned}
$$

If $\lambda$ is of type (W4), we can write $\lambda=\omega_{l_{1}}+\cdots+\omega_{l_{p}}+\omega_{l_{p+1}}+\cdots+$ $\omega_{l_{q-1}}+\Lambda_{n-1}$ with $1 \leqq l_{1} \leqq \cdots \leqq l_{p}<n<n+1=l_{p+1}=\cdots=l_{q-1}$. Then for $u_{k}=$\begin{tabular}{c|c}
\hline$t_{1}^{k}$ \\
\hline$\vdots$ \\
\hline$t_{l_{k}}^{k}$
\end{tabular}$\in B\left(\omega_{l_{k}}\right)(1 \leqq k<q)$ and $u_{q}=\left[\begin{array}{c}t_{1}^{q} \\
\vdots \vdots \\
\hline t_{n}^{q}\end{array} \in B_{\mathrm{sp}}^{(-)}\right.$, we denote $u_{1} \otimes \cdots \otimes u_{q}=$

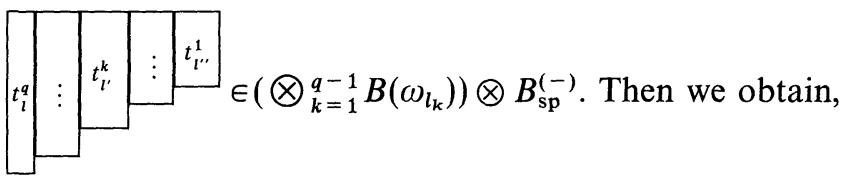

$$
B(\lambda)=\left\{w=t_{t^{a}} \vdots \vdots t_{l^{\prime}}: \vdots t_{l^{\prime \prime}}^{t^{1}} \in(\underbrace{q-1}_{k=1} B\left(\omega_{l_{k}}\right)) \otimes B_{\mathrm{sp}}^{(-)} ; \begin{array}{l}
w \text { satisfies the } \\
\text { following (1)-(4) }
\end{array}\right\}
$$

(1) $u_{k} \otimes u_{k+1} \in B\left(\omega_{l_{k}}+\omega_{l_{k+1}}\right)$ for any $k=1, \ldots, p-1$,

(2) $u_{p} \otimes u_{p+1} \in B\left(\omega_{l_{p}}+\omega_{n+1}\right)$,

(3) $u_{k} \otimes u_{k+1} \in B\left(2 \omega_{n+1}\right)$, for any $k=p+1, \ldots, q-2$,

(4) $u_{q-1} \otimes u_{q} \in B\left(\omega_{n+1}+\Lambda_{n-1}\right)$.

For $\lambda$ of type (W1)-(W3), we can obtain $B(\lambda)$ similarly. An element of $B(\lambda)$ is called a semi-standard $D$-tableau of shape $\lambda$.

\section{Generalized Young Diagrams}

It is well known that arbitrary finite-dimensional irreducible representations of $A_{n}$ and $C_{n}$ are characterized by Young diagrams. But it is not true for the $B_{n}$ and $D_{n}$ cases. In this section we shall introduce a "generalized Young diagram of type $\mathfrak{g}\left(\mathfrak{g}=A_{n}, B_{n}, C_{n}, D_{n}\right)$ " and characterize arbitrary finite-dimensional irreducible representations of $U_{q}(\mathfrak{g})$ in terms of "generalized Young diagrams."

3.1. Definition of generalized Young diagrams. For a sequence of half integers $l_{j}$ $(j=1,2, \ldots, n=$ rank of $\mathfrak{g})$, such that $l_{j}-l_{j+1} \in \mathbf{Z}_{\geqq 0}$, let $Y=\left(l_{1}, l_{2}, \ldots, l_{n}\right)$ mean a diagram which has $n$ rows and a length of the $j$-th row is $l_{j}$ (includes "-" length). We associate it with a weight $\sum_{j=1}^{n} l_{j} \varepsilon_{j}$. 
For example, if $Y=\left(l_{1}, l_{2}, \ldots, l_{n}\right)$ satisfies $l_{j} \in \mathbf{Z}_{\geqq 0}$, then $Y$ is an ordinary Young diagram. Now we define a "generalized Young diagram of type g."

Definition 3.1.1. Let $Y=\left(l_{1}, l_{2}, \ldots, l_{n}\right)$ be a diagram such that $l_{j}-l_{j+1} \in \mathbf{Z}_{\geqq 0}$.

(1) $\mathfrak{g}=A_{n}, C_{n}$-case: $Y=\left(l_{1}, l_{2}, \ldots, l_{n}\right)$ is a generalized Young diagram of type $A_{n}$ and $C_{n}$ if all $l_{j}$ are non-negative integers respectively.

(2) $\mathfrak{g}=B_{n}$-case: $Y=\left(l_{1}, l_{2}, \ldots, l_{n}\right)$ is a generalized Young diagram of type $B_{n}$ if all $l_{j}$ are non-negative half integers.

(3) $\mathrm{g}=D_{n}$-case: $Y=\left(l_{1}, l_{2}, \ldots, l_{n}\right)$ is a generalized Young diagram of type $D_{n}$ if all $l_{j}$ are half integers and $l_{1} \geqq l_{2} \geqq \cdots \geqq l_{n-1} \geqq\left|l_{n}\right|$.

Example 3.1.2. In the case of $g=D_{4}$, the generalized Young diagram $\left(\frac{5}{2}, \frac{3}{2}, \frac{3}{2},-\frac{1}{2}\right)$ of type $D_{4}$ is visualized as follows;

$$
\left(\frac{5}{2}, \frac{3}{2}, \frac{3}{2},-\frac{1}{2}\right)=\square .
$$

Here, "black box" means "-".

The following proposition is straightforward.

Proposition 3.1.3. Let $\mathscr{Y}$ be the set of generalized Young diagrams of type $\mathrm{g}=A_{n}, B_{n}$, $C_{n}$ and $D_{n}$ and $P_{+}$the set of dominant integral weights of $\mathrm{g}$. The map $\Psi: \mathscr{Y} \rightarrow \mathscr{P}_{+}$ defined by $Y=\left(l_{1}, \ldots, l_{n}\right) \mapsto \sum_{j=1}^{n} l_{j} \varepsilon_{j}$ gives an isomorphism from $\mathscr{Y}$ to $P_{+}$.

Remark 3.1.4. For $Y=\left(l_{1}, \ldots, l_{n}\right)$, the image by $\Psi$ is described as follows;

$$
\Psi(Y)= \begin{cases}\sum_{k=1}^{n-1}\left(l_{k}-l_{k+1}\right) \Lambda_{k}+l_{n} \Lambda_{n} & \text { if } \mathfrak{g}=A_{n}, C_{n}, \\ \sum_{k=1}^{n-1}\left(l_{k}-l_{k+1}\right) \Lambda_{k}+2 l_{n} \Lambda_{n} & \text { if } \mathfrak{g}=B_{n}, \\ \sum_{k=1}^{n-1}\left(l_{k}-l_{k+1}\right) \Lambda_{k}+\left(l_{n-1}+l_{n}\right) \Lambda_{n} & \text { if } \mathfrak{g}=D_{n} .\end{cases}
$$

Example 3.1.5. The image by $\Psi$ of the generalized Young diagram $\left(\frac{5}{2}, \frac{3}{2}, \frac{3}{2},-\frac{1}{2}\right)$ of type $D_{4}$ in Example 3.1 .2 is

$$
\frac{5}{2} \varepsilon_{1}+\frac{3}{2} \varepsilon_{2}+\frac{3}{2} \varepsilon_{3}-\frac{1}{2} \varepsilon_{4}=\Lambda_{1}+2 \Lambda_{3}+\Lambda_{4} \in P_{+} .
$$

3.2. Property of highest weight elements of $B(\lambda) \otimes B(\mu)$. The following proposition is a key for descriptions of a generalization of the Littlewood-Richardson rule.

Proposition 3.2.1. Let $\lambda$ and $\mu$ be dominant integral weights of g. For $u \in B(\lambda)$ and $v \in B(\mu)$, the following two conditions are equivalent;

(a) $\tilde{e}_{i}(u \otimes v)=0$ for any $i$.

(b) $\tilde{e}_{i} u=0$ and $\tilde{e}_{i}^{\left\langle h_{i}, \lambda\right\rangle+1} v=0$ for any $i$.

Proof. By Corollary 1.1.6, the condition (a) is equivalent to

$$
\varepsilon_{i}(u \otimes v)=\max \left(\varepsilon_{i}(u), \varepsilon_{i}(u)+\varepsilon_{i}(v)-\varphi_{i}(u)\right)=0 \quad \text { for any } i .
$$

The condition (b) is equivalent to

$$
\varepsilon_{i}(u)=0 \text { and } \varepsilon_{i}(v) \leqq\left\langle h_{i}, \lambda\right\rangle \text { for any } i .
$$


Note that by the definition of $\varphi_{i}(u)$ and $\varepsilon_{i}(u)$, we have

$$
\varphi_{i}(u)-\varepsilon_{i}(u)=\left\langle h_{i}, w t(u)\right\rangle \text {. }
$$

If $\varepsilon_{i}(u)=0$ for any $i$, then $w t(u)=\lambda$. Therefore, from (3.2.3), we obtain the equivalence of (3.2.1) and (3.2.2).

Q.E.D.

\section{Decomposition of $V_{Y} \otimes V_{\square}$}

In this section, for $\mathrm{g}=A_{n}, B_{n}, C_{n}$ and $D_{n}$, we shall give combinatorial descriptions for irreducible decomposition of $U_{q}(\mathrm{~g})$-module $V_{Y} \otimes V_{\square}$ with the help of crystal bases. Here, $Y$ is a generalized Young diagram of type $g$ and $V_{Y}$ is the irreducible $U_{q}(\mathfrak{g})$-module with highest weight $\Psi(Y)$. Of course, this result is well-known, but it is important that we can explain this in terms of crystal bases.

4.1. Lemmas. Let $Y=\left(l_{1}, l_{2}, \ldots, l_{n}\right)$ be a generalized Young diagram of type $\mathrm{g}$ and $u_{Y}$ the highest weight element of $B\left(V_{Y}\right)$. The following lemmas play an important role in this section.

Lemma 4.1.1. Let $\mathrm{g}$ be of type $A_{n}$. For a Young diagram $Y=\left(l_{1}, l_{2}, \ldots, l_{n}\right)$ and $j \in B\left(V_{\square}\right)(j=1,2, \ldots, n+1)$, the following (i) and (ii) are equivalent;

(i) $u_{Y} \otimes j$ is a highest weight element of $B\left(V_{Y} \otimes V_{\square}\right)$.

(ii) $l_{j-1}-l_{j}>0$.

Here, we set $l_{0}=\infty$ and $l_{n+1}=0$. (If $\mathfrak{g}=\mathfrak{g l}(n+1)$, we consider $Y$ has the $n+1$-th row.)

Proof. Let $\lambda$ be the weight of $Y$, i.e. $\lambda=\Psi(Y)$. By Proposition 3.2.1,

$u_{Y} \otimes j$ is a highest weight element if and only if $\varepsilon_{i}\left([j) \leqq\left\langle h_{i}, \lambda\right\rangle\right.$ for any $i$.

By the crystal graph (2.1.1) of the $U_{q}\left(A_{n}\right)$-module $V_{\square}$, we obtain,

$$
\varepsilon_{i}\left([)=\delta_{i, j-1}\right. \text {. }
$$

From (4.1.1) and (4.1.2), we obtain that $u_{Y} \otimes \square$ is always a highest weight element of $B\left(V_{Y} \otimes V_{\square}\right)$ and for $j=2, \ldots, n+1$,

$$
\begin{aligned}
& \varepsilon_{i}\left([ ) \leqq \langle h _ { i } , \lambda \rangle \text { for any } i \Leftrightarrow \varepsilon _ { j - 1 } \left([j) \leqq\left\langle h_{j-1}, \lambda\right\rangle\right.\right. \\
& \Leftrightarrow\left\langle h_{j-1}, \lambda\right\rangle \text { is positive } .
\end{aligned}
$$

Since $\left\langle h_{j-1}, \lambda\right\rangle=l_{j-1}-l_{j}$, we obtain the equivalence of (i) and (ii).

The proofs of the following lemmas are quite similar to that of Lemma 4.1.1, so we omit them.

Lemma 4.1.2. Let $\mathfrak{g}$ be of type $C_{n}$ and $Y=\left(l_{1}, l_{2}, \ldots, l_{n}\right)$ be a Young diagram.

(1) For $j \in B\left(V_{\square}\right)(j=1,2, \ldots, n), u_{Y} \otimes j$ is a highest weight element of $B\left(V_{Y} \otimes V_{\square}\right)$ if and only if $l_{j-1}-l_{j}>0\left(l_{0}=\infty\right)$.

(2) For $\bar{j} \in B\left(V_{\square}\right)(j=1,2, \ldots, n), u_{Y} \otimes \bar{j}$ is a highest weight element of $B\left(V_{Y} \otimes V_{\square}\right)$ if and only if $l_{j}-l_{j+1}>0\left(l_{n+1}=0\right)$. 
Lemma 4.1.3. Let $\mathfrak{g}$ be of type $B_{n}$ and $Y=\left(l_{1}, l_{2}, \ldots, l_{n}\right)$ a generalized Young diagram of type $B_{n}$.

(1) For $j \in B\left(V_{\square}\right)(j=1,2, \ldots, n), u_{Y} \otimes j$ is a highest weight element of $B\left(V_{Y} \otimes V_{\square}\right)$ if and only if $l_{j-1}-l_{j}>0$.

(2) For $\bar{j} \in B\left(V_{\square}\right)(j=1,2, \ldots, n-1), u_{Y} \otimes \bar{j}$ is a highest weight element of $B\left(V_{Y} \otimes V_{\square}\right)$ if and only if $l_{j}-l_{j+1}>0$.

(3) For $0 \in B\left(V_{\square}\right), u_{Y} \otimes 0$ is a highest weight element of $B\left(V_{Y} \otimes V_{\square}\right)$ if and only if $l_{n}>0$.

(4) For $\overline{\bar{n}} \in B\left(V_{\square}\right), u_{Y} \otimes \bar{n}$ is a highest weight element of $B\left(V_{Y} \otimes V_{\square}\right)$ if and only if $l_{n} \geqq 1$.

Note that for a generalized Young diagram of type $B_{n}$, the condition $l_{n}>0$ in (3) is not equivalent to the condition $l_{n} \geqq 1$ in (4).

Lemma 4.1.4. Let $g$ be of type $D_{n}$ and $Y=\left(l_{1}, l_{2}, \ldots, l_{n}\right)$ a generalized Young diagram of type $D_{n}$.

(1) For $j \in B\left(V_{\square}\right)(j=1,2, \ldots, n), u_{Y} \otimes j$ is a highest weight element of $B\left(V_{Y} \otimes V_{\square}\right)$ if and only if $l_{j-1}-l_{j}>0$.

(2) For $\bar{j} \in B\left(V_{\square}\right)(j=1,2, \ldots, n-2), u_{Y} \otimes \bar{j}$ is a highest weight element of $B\left(V_{Y} \otimes V_{\square}\right)$ if and only if $l_{j}-l_{j+1}>0$.

(3) For $\bar{n} \in B\left(V_{\square}\right), u_{Y} \otimes \bar{n}$ is a highest weight element of $B\left(V_{Y} \otimes V_{\square}\right)$ if and only if $l_{n-1} \neq-l_{n}$.

(4) For $\sqrt{n-1} \in B\left(V_{\square}\right), u _ { Y } \otimes \longdiv { n - 1 }$ is a highest weight element of $B\left(V_{Y} \otimes V_{\square}\right)$ if and only if $l_{n-1}>\left|l_{n}\right|$.

4.2. For a generalized Young diagram $Y=\left(l_{1}, l_{2}, \ldots, l_{n}\right)$ of type $\mathfrak{g}$, we shall define the following notations.

$$
\begin{aligned}
(Y \leftarrow j): & \left(l_{1}, \ldots, l_{j}+1, \ldots, l_{n}\right) \text { for } j=1, \ldots, n, \\
(Y \leftarrow \bar{j}):=\left(l_{1}, \ldots, l_{j}-1, \ldots, l_{n}\right) \text { for } j=1, \ldots, n, & \\
(Y \leftarrow n+1): & = \begin{cases}\left(l_{1}-1, l_{2}-1, \ldots, l_{n}-1\right) & \mathrm{g}=A_{n}, \\
\left(l_{1}, l_{2}, \ldots, l_{n}, l_{n+1}+1\right) & \mathrm{g}=\mathrm{gl}(n+1),\end{cases} \\
(Y \leftarrow 0): & = \begin{cases}\left(l_{1}, \ldots, l_{n}\right) & \text { if } l_{n}>0, \\
\left(l_{1}, \ldots, l_{n-1},-\infty\right) & \text { if } l_{n}=0 .\end{cases}
\end{aligned}
$$

Note that the case $l_{n}=0$ in (4.2.4) implies that $(Y \leftarrow 0)$ is not an element of $\mathscr{Y}$ and for $\mathfrak{g}=\mathfrak{g l}(n+1)$, we consider that $Y$ has $n+1$ rows.

Proposition 4.2.1. Let $Y=\left(l_{1}, l_{2}, \ldots, l_{n}\right)$ be a generalized Young diagram of type $\mathrm{g}$ and $V_{Y}$ a finite-dimensional irreducible $U_{q}(\mathrm{~g})$-module associated to $Y$. Then we have

$$
V_{Y} \otimes V_{\square} \cong \begin{cases}\bigoplus_{j=1}^{n+1} V_{(Y \leftarrow j)} & \text { if } \mathfrak{g}=A_{n} \text { or } \mathfrak{g l}(n+1), \\ \bigoplus_{j=0}^{n} V_{(Y \leftarrow j)} \oplus \bigoplus_{j=1}^{n} V_{(Y \leftarrow \bar{j})} & \text { if } \mathfrak{g}=B_{n}, \\ \bigoplus_{j=1}^{n} V_{(Y \leftarrow j)} \oplus \bigoplus_{j=1}^{n} V_{(Y \leftarrow \bar{j})} & \text { if } \mathfrak{g}=C_{n}, D_{n} .\end{cases}
$$

Here if $Y$ is not a generalized Young diagram, then $V_{Y}$ means the 0 -dimensional vector space. 
Proof. By Corollary 1.1.4, it suffices to determine all the highest weight elements of $B\left(V_{Y} \otimes V_{\square}\right)$. By Proposition 3.2.1, if $u \otimes v \in B\left(V_{Y} \otimes V_{\square}\right)$ is a highest weight element, then we have $u=u_{Y}$. Hence, by Lemma 4.1.1-4.1.4 we have already known all the highest weight elements of $B\left(V_{Y} \otimes V_{\square}\right)$ in terms of $l_{j}$ 's. We shall restate this in terms of generalized Young diagrams.

(i) $\mathfrak{g}=A_{n}(\mathfrak{g l}(n+1))$-case

The condition $l_{j-1}-l_{j}>0$ in Lemma 4.1.1 is equivalent to the condition that $(Y \leftarrow j)$ remains a Young diagram. Hence $u_{Y} \otimes j$ is a highest weight element of $B\left(V_{Y} \otimes V_{\square}\right)$ if and only if $(Y \leftarrow j)$ is a Young diagram. It remains to note

$$
w t\left(u_{Y} \otimes j\right)=\left(\sum_{k=1}^{n} l_{k} \varepsilon_{k}\right)+\varepsilon_{j}=w t(Y \leftarrow j) .
$$

(If $\mathrm{g}=A_{n}$, since $\varepsilon_{1}+\cdots+\varepsilon_{n+1}=0$, it is true for the case $j=n+1$.) Hence we obtain the case $A_{n}(\mathfrak{g l}(n+1))$-case.

(ii) $\mathfrak{g}=C_{n}$-case

Similarly to the $A_{n}$-case, by Lemma 4.1.2.(1), $u_{Y} \otimes j$ is a highest weight element of $B\left(V_{Y} \otimes V_{\square}\right)$ if and only if $(Y \leftarrow j)$ remains a Young diagram. It remains to note that $w t(Y \leftarrow j)=w t\left(u_{Y} \otimes[j)\right.$.

The condition $l_{j}-l_{j+1}>0$ in Lemma 4.1.2.(2) is equivalent to the condition that $(Y \leftarrow \bar{j})$ remains a Young diagram. Hence $u_{Y} \otimes \bar{j}$ is a highest weight element of $B\left(V_{Y} \otimes V_{\square}\right)$ if and only if $(Y \leftarrow \bar{j})$ is a Young diagram. It remains to note that

$$
w t\left(u_{Y} \otimes \overline{\bar{j}}\right)=\left(\sum_{k=1}^{n} l_{k} \varepsilon_{k}\right)-\varepsilon_{j}=w t(Y \leftarrow \bar{j}) .
$$

Therefore we get the $C_{n}$-case.

(iii) $\mathfrak{g}=B_{n}$-case

Similarly to the $C_{n}$-case, by Lemma 4.1.3(1) and (2), $u_{Y} \otimes{ }_{j} \in B\left(V_{Y} \otimes V_{\square}\right)$ $(j=1, \ldots, n)$ satisfies the highest weight condition if and only if $(Y \leftarrow j)$ is a generalized Young diagram of type $B_{n}$ and $u_{Y} \otimes \bar{j} \in B\left(V_{Y} \otimes V_{\square}\right)$ $(j=1, \ldots, n-1)$ satisfies the highest condition if and only if $(Y \leftarrow \bar{j})$ is a generalized Young diagram of type $B_{n}$. We have $w t\left(u_{Y} \otimes j\right)=w t(Y \leftarrow j)$ and $w t\left(u_{Y} \otimes \bar{j}\right)=w t(Y \leftarrow \bar{j})$.

By Lemma 4.1.3.(3), $u_{Y} \otimes 0$ is a highest weight element of $B\left(V_{Y} \otimes V_{\square}\right)$ if and only if $(Y \leftarrow 0)$ remains a generalized Young diagram of type $B_{n}$. Both $Y \otimes 0$ and $Y$ have the same weight $\sum_{k=1}^{n} l_{k} \varepsilon_{k}$.

By Lemma 4.1.3.(4), $u_{Y} \otimes \bar{n} \in B\left(V_{Y} \otimes V_{\square}\right)$ is a highest weight element if and only if $l_{n} \geqq 1$. Here, the condition $l_{n} \geqq 1$ is equivalent to the condition that $(Y \leftarrow \bar{n})$ remains a generalized Young diagram. We have

$$
w t(Y \otimes \bar{n})=\left(\sum_{k=1}^{n} l_{k} \varepsilon_{k}\right)-\varepsilon_{n}=w t(Y \leftarrow \bar{n}) .
$$

By these results, we obtain the $B_{n}$-case.

(iv) $\mathfrak{g}=D_{n}$-case

Similarly to the $B_{n}$-case and the $C_{n}$-case, by Lemma 4.1.4.(1) and (2), if $u \otimes j$ $(j=1, \ldots, n)$ (respectively $u \otimes \bar{j}(j=1, \ldots, n-2)) \in B\left(V_{Y} \otimes V_{\square}\right)$ satisfies the highest weight condition if and only if $(Y \leftarrow j)$ (respectively $(Y \leftarrow \bar{j}))$ is a generalized 
Young diagram of type $D_{n}$. We have $w t\left(u_{Y} \otimes j\right)=w t(Y \leftarrow j)$ and $w t\left(u_{Y} \otimes \overline{\bar{j}}\right)=w t(Y \leftarrow \bar{j})$. By Lemma 4.1.4.(3), $u_{Y} \otimes \overline{\bar{n}}$ is a highest weight element of $B\left(V_{Y} \otimes V_{\square}\right)$ if and only if $l_{n} \neq-l_{n-1}$. By the definition of generalized Young diagram of type $D_{n}$, the condition $l_{n} \neq-l_{n-1}$ is equivalent to the condition that $(Y \leftarrow \bar{n})=\left(l_{1}, \ldots, l_{n-1}, l_{n}-1\right)$ remains a generalized Young diagram. We have

$$
w t(Y \otimes \bar{n})=\left(\sum_{k=1}^{n} l_{k} \varepsilon_{k}\right)-\varepsilon_{n}=w t(Y \leftarrow \bar{n}) .
$$

By Lemma 4.1.4.(4), $u _ { Y } \otimes \longdiv { n - 1 }$ is a highest weight element of $B\left(V_{Y} \otimes V_{\square}\right)$ if and only if $l_{n-1}>\left|l_{n}\right|$. By the definition of generalized Young diagram of type $D_{n}$, the condition $l_{n-1}>\left|l_{n}\right|$ is equivalent to the condition that $(Y \leftarrow \overline{n-1})$ remains a generalized Young diagram of type $D_{n}$. It remains to note

$$
w t(Y \otimes \overline{n-1})=\left(\sum_{k=1}^{n} l_{k} \varepsilon_{k}\right)-\varepsilon_{n-1}=w t(Y \leftarrow \overline{n-1}) .
$$

Thus, we obtain the $D_{n}$-case.

Q.E.D.

Example 4.2.2. For $\mathrm{g}=B_{3}$ and $Y=(2,2,1)=\square$, we consider $V_{Y} \otimes V_{\square}$. We have $\Psi(Y)=2 \varepsilon_{1}+2 \varepsilon_{2}+\varepsilon_{3}=\Lambda_{2}+2 \Lambda_{3}$ and $B\left(V_{\square}\right)=\{\square, \quad, 2, \quad 3,[0, \overline{3}$, $\overline{2}, \overline{1}\}$.

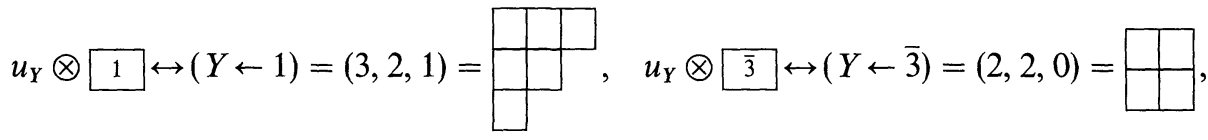

$$
\begin{aligned}
& u_{Y} \otimes 2=(Y \leftarrow 2)=(2,3,1) \times, \\
& u_{Y} \otimes \overline{2} \leftrightarrow(Y \leftarrow \overline{2})=(2,1,1)=\square \\
& u_{Y} \otimes 3 \leftrightarrow(Y \leftarrow 3)=(2,2,2)=\square, \quad u_{Y} \otimes \overline{1} \leftrightarrow(Y \leftarrow \overline{1})=(1,2,1) \times, \\
& u_{Y} \otimes \square \leftrightarrow(Y \leftarrow 0)=(2,2,1)=\square .
\end{aligned}
$$

Therefore, we get

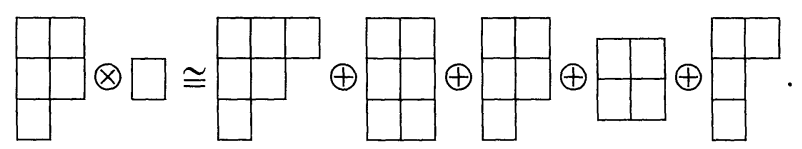

Hence,

$$
\begin{gathered}
V\left(\Lambda_{2}+2 \Lambda_{3}\right) \otimes V_{\square} \cong V\left(\Lambda_{1}+\Lambda_{2}+2 \Lambda_{3}\right) \oplus V\left(4 \Lambda_{3}\right) \oplus V\left(\Lambda_{2}+2 \Lambda_{3}\right) \\
\oplus V\left(2 \Lambda_{2}\right) \oplus V\left(\Lambda_{1}+2 \Lambda_{3}\right) .
\end{gathered}
$$


Example 4.2.3. For $\mathfrak{g}=D_{4}$ and $Y=(2,2,2,-1)=\square$ ( $\square$ means "-"), we
consider $V_{\mathrm{Y}} \otimes V_{\square}$. We have $\Psi(Y)=2 \varepsilon_{1}+2 \varepsilon_{2}+2 \varepsilon_{3}-\varepsilon_{4}=3 \Lambda_{3}+\Lambda_{4}$ and

$$
\begin{aligned}
& B\left(V_{\square}\right)=\{\overline{1}, \overline{2}, \overline{3}, \overline{4}, \overline{4}, \overline{3}, \overline{2}, \overline{1}\} \\
& u_{Y} \otimes 1, \leftrightarrow(Y \leftarrow 1)=(3,2,2,-1)=\begin{array}{|l|l|}
\hline & \\
\hline & \\
\hline
\end{array} \\
& u_{Y} \otimes 2 \leftrightarrow(Y \leftarrow 2)=(2,3,2,-1) \times, \\
& u_{Y} \otimes 3 \leftrightarrow(Y \leftarrow 3)=(2,2,3,-1) \times, \\
& u_{Y} \otimes 4 \leftrightarrow(Y \leftarrow 4)=(2,2,2,0)=\square, \\
& u_{Y} \otimes \overline{4} \leftrightarrow(Y \leftarrow \overline{4})=(2,2,2,-2)=\square \\
& u_{Y} \otimes \overline{\overline{3}} \leftrightarrow(Y \leftarrow \overline{3})=(2,2,1,-1)=\square, \\
& u_{Y} \otimes \overline{2} \leftrightarrow(Y \leftarrow \overline{2})=(2,1,2,-1) \times, \\
& u_{Y} \otimes \overline{1} \leftrightarrow(Y \leftarrow \overline{1})=(1,2,2,-1) \times .
\end{aligned}
$$

Then, we obtain (we omit $V$ )
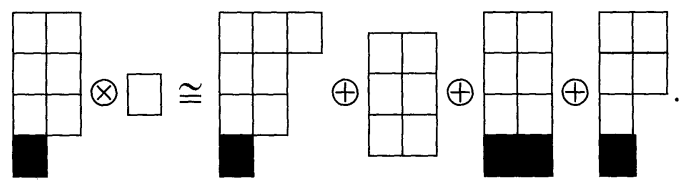

Hence,

$$
\begin{gathered}
V\left(3 \Lambda_{3}+\Lambda_{4}\right) \otimes V_{\square} \cong V\left(\Lambda_{1}+3 \Lambda_{3}+\Lambda_{4}\right) \oplus V\left(2 \Lambda_{3}+2 \Lambda_{4}\right) \\
\oplus V\left(4 \Lambda_{3}\right) \oplus V\left(\Lambda_{2}+2 \Lambda_{3}\right) .
\end{gathered}
$$




\section{Decomposition of $V_{Y} \otimes V_{\text {sp }}$}

For $\mathfrak{g}=B_{n}$ and $D_{n}$, there exist "spin representations." In this section we shall give combinatorial descriptions for irreducible decomposition of $U_{q}(\mathfrak{g})$-module $V_{Y} \otimes V_{\text {sp }}$ by use of crystal bases.

5.1. Decomposition of $U_{q}\left(B_{n}\right)$-module $V_{\mathrm{Y}} \otimes V_{\mathrm{sp}}$. We have already introduced the spin representation $V_{\mathrm{sp}}$ of $U_{q}\left(B_{n}\right)$ in 2.3. In this section we introduce another description of $V_{\mathrm{sp}}([\mathrm{Re}])$.

First we set $B_{\mathrm{sp}}=\left\{v=\left(i_{1}, i_{2}, \ldots, i_{n}\right) ; i_{j}= \pm\right\}, V_{\mathrm{sp}}=\bigoplus_{v \in \boldsymbol{B}_{\mathrm{sp}}} \mathbf{Q}(q) v$ and $L_{\mathrm{sp}}=\bigoplus_{v \in B_{\mathrm{sp}}} A v$. For $v=\left(i_{1}, i_{2}, \ldots, i_{n}\right) \in B_{\mathrm{sp}}$, if we define the actions of generators and the operators $\tilde{e}_{i}$ and $\tilde{f}_{i}$ as follows:

$$
\begin{aligned}
& q^{h} v=q^{\langle h, w t(v)\rangle} v, \text { where } w t(v)=\frac{1}{2} \sum_{j=1}^{n} i_{j} \varepsilon_{j} \text { for } v=\left(i_{1}, \ldots, i_{n}\right) \\
& e_{j} v=\tilde{e}_{j} v=\left\{\begin{array}{c}
\left(i_{1}, \ldots,+{ }^{j}, \stackrel{j+1}{-}, \ldots, i_{n}\right) \quad \begin{array}{l}
i_{j}=- \text { and } i_{j+1}=+ \\
0
\end{array} \text { otherwise },
\end{array}\right. \\
& f_{j} v=\tilde{f}_{j} v=\left\{\begin{array}{c}
\left(i_{1}, \ldots, \frac{j}{-},+1+\ldots, i_{n}\right) \quad \begin{array}{l}
i_{j}=+ \text { and } i_{j+1}=- \\
0
\end{array} \text { otherwise },
\end{array}\right. \\
& \text { for } j=1, \ldots, n-1 \text {, and } \\
& e_{n} v=\tilde{e}_{n} v=\left\{\begin{array}{cl}
\left(i_{1}, \ldots, i_{n-1}, \stackrel{n}{+}\right) & i_{n}=-, \\
0 & \text { otherwise },
\end{array}\right. \\
& f_{n} v=\tilde{f}_{n} v=\left\{\begin{array}{cl}
\left(i_{1}, \ldots, i_{n-1}, \stackrel{n}{-}\right) & i_{n}=+, \\
0 & \text { otherwise }
\end{array}\right.
\end{aligned}
$$

then $V_{\mathrm{sp}} \cong V\left(\Lambda_{n}\right)$ and $\left(L_{\mathrm{sp}}, B_{\mathrm{sp}}\right)$ is a crystal base of $V_{\mathrm{sp}}$.

Note that the correspondence of this description and the description in 2.3 is given by:

$$
\text { " }+ \text { " in the } j \text {-th row } \leftrightarrow j \text { and "-" in the } j \text {-th row } \leftrightarrow \bar{j}
$$

Now, for a generalized Young diagram $Y=\left(l_{1}, \ldots, l_{n}\right)$ and $v=\left(i_{1}, \ldots, i_{n}\right) \in B_{\text {sp }}$ we define

$$
(Y+v):=\left(l_{1}+\frac{1}{2} i_{1}, l_{2}+\frac{1}{2} i_{2}, \ldots, l_{n}+\frac{1}{2} i_{n}\right) .
$$

The following lemma plays a similar role to Lemmas 4.1.1-4.1.4 in Sect. 4.

Lemma 5.1.1. Let $Y=\left(l_{1}, l_{2}, \ldots, l_{n}\right)$ be a generalized Young diagram of type $B_{n}$ and $u_{Y}$ the highest weight element of $B\left(V_{Y}\right)$. For $v=\left(i_{1}, i_{2}, \ldots, i_{n}\right) \in B_{\mathrm{sp}}$ the following two statements are equivalent,

(a) $u_{Y} \otimes v$ is a highest weight element of $B\left(V_{Y} \otimes V_{\mathrm{sp}}\right)$.

(b) $(Y+v)$ is a generalized Young diagram of type $B_{n}$. 
Proof. Let $\lambda=\sum_{k=1}^{n} l_{k} \varepsilon_{k}$ be the weight of $Y$. By Proposition 3.2.1, $u_{Y} \otimes v$ is a highest weight element of $B\left(V_{Y} \otimes V_{\text {sp }}\right)$ if and only if $\varepsilon_{j}(v) \leqq\left\langle h_{j}, \lambda\right\rangle$ for any $j$. Therefore it is enough to show that $(Y+v)=\left(l_{1}+\frac{1}{2} i_{1}, l_{2}+\frac{1}{2} i_{2}, \ldots, l_{n}+\frac{1}{2} i_{n}\right)$ is not a generalized Young diagram of type $B_{n}$ if and only if $\left.\varepsilon_{j}(v)\right\rangle\left\langle h_{j}, \lambda\right\rangle$ for some $j$. By (5.1.2) and (5.1.4), we have $\varepsilon_{j}(v) \leqq 1$ for any $j$.

First we assume that $\left.\varepsilon_{j}(v)\right\rangle\left\langle h_{j}, \lambda\right\rangle$ for some $j$. Then we have

$$
\left\langle h_{j}, \lambda\right\rangle=0 \quad \text { and } \quad \varepsilon_{j}(v)=1
$$

If $j \neq n$, then from (5.1.2) and (5.1.6) we have

$$
\left\langle h_{j}, \lambda\right\rangle=0, \quad i_{j}=- \text { and } i_{j+1}=+.
$$

Since $\left\langle h_{j}, \lambda\right\rangle=l_{j}-l_{j+1}=0$, we obtain

$$
l_{j}+\frac{1}{2} i_{j}=l_{j}-\frac{1}{2}<l_{j+1}+\frac{1}{2}=l_{j+1}+\frac{1}{2} i_{j+1} .
$$

This implies that $(Y+v)$ is not a generalized Young diagram of type $B_{n}$.

If $j=n$, then (5.1.4) and (5.1.6) imply

$$
\left\langle h_{n}, \lambda\right\rangle=0 \text { and } i_{n}=- \text {. }
$$

The condition $\left\langle h_{n}, \lambda\right\rangle=0$ is equivalent to $l_{n}=0$. Therefore we have

$$
l_{n}+\frac{1}{2} i_{n}=-\frac{1}{2}<0 \text {. }
$$

This implies that $(Y+v)$ does not satisfy the condition of a generalized Young diagram of type $B_{n}$ in Definition 3.3.1 (2).

Next we assume that for a generalized Young diagram $Y=\left(l_{1}, l_{2}, \ldots, l_{n}\right)$ of type $B_{n}$ and $v=\left(i_{1}, i_{2}, \ldots, i_{n}\right) \in B_{\text {sp }},(Y+v)=\left(l_{1}+\frac{1}{2} i_{1}, l_{2}+\frac{1}{2} i_{2}, \ldots, l_{n}+\frac{1}{2} i_{n}\right)$ is not a generalized Young diagram of type $B_{n}$. One of the following cases can occur,

$$
\begin{aligned}
& l_{j}+\frac{1}{2} i_{j}<l_{j+1}+\frac{1}{2} i_{j+1} \quad \text { for some } j \neq n, \\
& l_{n}+\frac{1}{2} i_{n}<0
\end{aligned}
$$

Note that $(Y+v)$ always satisfies $\left(l_{j}+\frac{1}{2} i_{j}\right)-\left(l_{j+1}+\frac{1}{2} i_{j+1}\right) \in \mathbf{Z}$. get

In the case (5.1.11), from the condition $l_{j}-l_{j+1} \in \mathbf{Z}_{\geqq 0}$ in Definition 3.3.1.(2), we

$$
l_{j}=l_{j+1}, \quad i_{j}=- \text { and } i_{j+1}=+.
$$

This implies that $\left\langle h_{j}, \lambda\right\rangle=0$ and $\varepsilon_{j}(v)=1$. Hence, $\tilde{e}_{j}\left(u_{Y} \otimes v\right) \neq 0$.

In the case (5.1.12), we have

$$
l_{n}=0 \text { and } i_{n}=- \text {. }
$$

This implies that $\left\langle h_{n}, \lambda\right\rangle=0$ and $\varepsilon_{n}(v)=1$. Hence, $\tilde{e}_{n}\left(u_{Y} \otimes v\right) \neq 0$. Thus we get the equivalence of (a) and (b).

Q.E.D. 
Proposition 5.1.2. Let $Y=\left(l_{1}, l_{2}, \ldots, l_{n}\right)$ be a generalized Young diagram of type $B_{n}$. For $U_{q}\left(B_{n}\right)$-modules $V_{Y}$ and $V_{\mathrm{sp}}$, we obtain;

$$
V_{Y} \otimes V_{\mathrm{sp}} \cong \bigoplus_{v=\left(i_{1}, \ldots, i_{n}\right) \in B_{s p}} V_{(Y+v)} .
$$

Here if $Y$ is not a generalized Young diagram, $V_{Y}$ means the 0 -dimensional vector space.

Proof. By Proposition 3.2.1 and Lemma 5.1.1, $u \otimes v \in B\left(V_{Y} \otimes V_{\mathrm{sp}}\right)$ satisfies the highest weight condition if and only if $u=u_{Y}$ and $(Y+v)$ is a generalized Young diagram of type $B_{n}$. Since $v=\left(i_{1}, i_{2}, \ldots, i_{n}\right)$ has weight $\frac{1}{2} \sum_{k=1}^{n} i_{k} \varepsilon_{k}$,

$$
w t\left(u_{Y} \otimes v\right)=\sum_{k=1}^{n}\left(l_{k}+\frac{1}{2} i_{k}\right) \varepsilon_{k}=w t(Y+v) .
$$

By Corollary 1.1.4, we obtain the desired result.

Q.E.D.

$$
\begin{aligned}
& \text { Example 5.1.3. For } \mathfrak{g}=B_{3} \text { and } Y=\left(\frac{3}{2}, \frac{3}{2}, \frac{1}{2}\right)=\square\left(=\Lambda_{2}+\Lambda_{3}\right) \\
& \qquad B_{\mathrm{sp}}=B\left(\Lambda_{3}\right)=\left\{\begin{array}{l}
(+,+,+),(+,+,-),(+,-,+),(-,+,+), \\
(+,-,-),(-,+,-),(-,-,+),(-,-,-) .
\end{array}\right\}
\end{aligned}
$$

$$
\begin{array}{ll}
u_{Y} \otimes(+,+,+) \leftrightarrow(2,2,1)=\square & u_{Y} \otimes(+,-,-) \leftrightarrow(2,1,0)=\square \\
u_{Y} \otimes(+,+,-) \leftrightarrow(2,2,0)=\square & u_{Y} \otimes(-,+,-) \leftrightarrow(1,2,0) \times \\
\hline \square & u_{Y} \otimes(-,-,+) \leftrightarrow(1,1,1)=\square \\
u_{Y} \otimes(+,-,+) \leftrightarrow(2,1,1)=\square & \\
u_{Y} \otimes(-,+,+) \leftrightarrow(1,2,1)=\times & u_{Y} \otimes(-,-,-) \leftrightarrow(1,1,0)=\square
\end{array}
$$

Then we get (we omit $V$ )

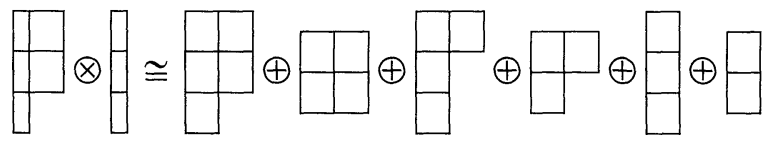

Hence,

$$
\begin{gathered}
V\left(\Lambda_{2}+\Lambda_{3}\right) \otimes V_{\mathrm{sp}} \cong \\
V\left(\Lambda_{2}+2 \Lambda_{3}\right) \oplus V\left(2 \Lambda_{2}\right) \oplus V\left(\Lambda_{1}+2 \Lambda_{3}\right) \\
V\left(\Lambda_{1}+\Lambda_{2}\right) \oplus V\left(2 \Lambda_{3}\right) \oplus V\left(\Lambda_{2}\right) .
\end{gathered}
$$

5.2. Decomposition of $U_{q}\left(D_{n}\right)$ module $V_{Y} \otimes V_{\mathrm{sp}}^{(+)}$and $V_{Y} \otimes V_{\mathrm{sp}}^{(-)}$. We have already introduced the two spin representations $V_{\mathrm{sp}}^{( \pm)}$of $U_{q}\left(D_{n}\right)$ in 2.4 . 
Similarly to the $B_{n}$-case we shall give another description of $V_{\mathrm{sp}}^{( \pm)}([\mathrm{Re}])$. First we set

$$
\begin{aligned}
B_{\mathrm{sp}}^{(+)}\left(\text {respectively } B_{\mathrm{sp}}^{(-)}\right) & =\left\{v=\left(i_{1}, \ldots, i_{n}\right) ;\right. \\
i_{j} & \left.= \pm, i_{1} \ldots i_{n}=+(\text { respectively }-)\right\}, \\
V_{\mathrm{sp}}^{( \pm)} & =\bigoplus_{v \in B_{\mathrm{sp}}^{( \pm)}} \mathbf{Q}(q) v \text { and } L_{\mathrm{sp}}^{( \pm)}=\bigoplus_{v \in B_{\mathrm{sp}}^{( \pm)}} A v,
\end{aligned}
$$

and if we define the actions of generators on $V_{\mathrm{sp}}^{( \pm)}$and the operators $\tilde{e}_{i}$ and $\tilde{f}_{i}$ on $L_{\mathrm{sp}}^{( \pm)}$as follows, for $v=\left(i_{1}, \ldots, i_{n}\right)$,

$$
\begin{aligned}
& q^{h} v=q^{\langle h, w t(v)\rangle} v, \text { where } w t(v)=\frac{1}{2} \sum_{j=1}^{n} i_{j} \varepsilon_{j}, \\
& e_{j} v=\tilde{e}_{i} v=\left\{\begin{array}{cl}
\left(i_{1}, \ldots,+{ }^{j+1}, \ldots, i_{n}\right) \quad & i_{j}=- \text { and } i_{j+1}=+, \\
0 & \text { otherwise },
\end{array}\right. \\
& f_{j} v=\tilde{f}_{i} v=\left\{\begin{array}{cl}
\left(i_{1}, \ldots, \frac{j}{-},+1\right. \\
0
\end{array}\right. \\
& (j=1, \ldots, n-1) \\
& e_{n} v=\tilde{e}_{n} v=\left\{\begin{array}{cc}
\left(i_{1}, \ldots, \begin{array}{cc}
n-1 & n \\
+, & +
\end{array}\right) & i_{n-1}=- \text { and } i_{n}=- \\
0 & \text { otherwise }
\end{array}\right.
\end{aligned}
$$

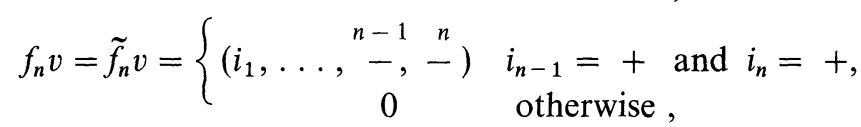

then $V_{\mathrm{sp}}^{(+)} \cong V\left(\Lambda_{n}\right), V_{\mathrm{sp}}^{(-)} \cong V\left(\Lambda_{n-1}\right)$ and $\left(L_{\mathrm{sp}}^{( \pm)}, B_{\mathrm{sp}}^{( \pm)}\right)$is a crystal base of $V_{\mathrm{sp}}^{( \pm)}$respectively. Note that the correspondence between this description and the description in 2.4 is given by,

$$
\text { " }+ \text { " in the } j \text {-th row } \leftrightarrow j \text { and "_" in the } j \text {-th row } \leftrightarrow \bar{j} .
$$

Lemma 5.2.1. Let $Y=\left(l_{1}, \ldots, l_{n}\right)$ be a generalized Young diagram of type $D_{n}$ and $u_{Y}$ the highest weight element of $B\left(V_{Y}\right)$. For $u_{Y}$ and $v=\left(i_{1}, \ldots, i_{n}\right) \in B_{\mathrm{sp}}^{( \pm)}$, the following two statements are equivalent;

(a) $u_{Y} \otimes v$ is a highest weight element of $B\left(V_{Y} \otimes V_{\mathrm{sp}}^{( \pm)}\right)$.

(b) $(Y+v)=\left(l_{1}+\frac{1}{2} i_{1}, l_{2}+\frac{1}{2} i_{2}, \ldots, l_{n}+\frac{1}{2} i_{n}\right)$ is a generalized Young diagram of type $D_{n}$.

The proof of this lemma is quite similar to that of Lemma 5.1.1, so we omit it. Proposition 5.2.2. Let $Y$ be a generalized Young diagram of type $D_{n}$. For $U_{q}\left(D_{n}\right)$ modules $V_{Y}$ and $V_{\mathrm{sp}}^{( \pm)}$, we obtain;

$$
\begin{aligned}
& V_{Y} \otimes V_{\mathrm{sp}}^{(+)} \cong \underset{v=\left(i_{1}, \ldots, i_{n}\right) \in B_{\mathrm{sp}}^{(+)}}{\bigoplus} V_{(Y+v)}, \\
& V_{Y} \otimes V_{\mathrm{sp}}^{(-)} \cong \underset{v=\left(i_{1}, \ldots, i_{n}\right) \in B_{\mathrm{sp}}^{(-)}}{\bigoplus} V_{(Y+v)},
\end{aligned}
$$

where if $Y$ is not a generalized Young diagram of type $D_{n}, V_{Y}$ means the 0 -dimensional vector space. 
Proof. By Proposition 3.2.1 and Lemma 5.2.1, $u \otimes v \in B\left(V_{Y} \otimes V_{\mathrm{sp}}^{( \pm)}\right)$satisfies the highest weight condition if and only if $u=u_{Y}$ and $(Y+v)$ is a generalized Young diagram of type $D_{n}$. The weight of $v=\left(i_{1}, i_{2}, \ldots, i_{n}\right)$ is $\frac{1}{2} \sum_{j=1}^{n} i_{j} \varepsilon_{j}$. Hence

$$
w t\left(u_{Y} \otimes v\right)=\sum_{k=1}^{n}\left(l_{k}+\frac{1}{2} i_{k}\right) \varepsilon_{k}=w t(Y+v) .
$$

Thus, by Corollary 1.1.4, we obtain the desired result.

Example 5.2.3. For $\mathfrak{g}=D_{4}$, we consider the decomposition $V\left(\Lambda_{1}+2 \Lambda_{3}\right) \otimes V_{\mathrm{sp}}^{(+)}$. $\Lambda_{1}+2 \Lambda_{3}$ corresponds to the generalized Young diagram $(2,1,1,-1)$.

We set

We have

$$
\begin{aligned}
Y= & (2,1,1,-1)=\square \text { and } B_{\mathrm{sp}}^{(+)}=B\left(\Lambda_{4}\right) \\
& =\left\{\begin{array}{ll}
(+,+,+,+), & (+,-,-,+) \\
(+,+,-,-), & (-,+,-,+) \\
(+,-,+,-), & (-,-,+,+) \\
(-,+,+,-), & (-,-,-,-)
\end{array}\right\} .
\end{aligned}
$$

$$
\begin{gathered}
u_{Y} \otimes(+,+,+,+) \leftrightarrow\left(\frac{5}{2}, \frac{3}{2}, \frac{3}{2},-\frac{1}{2}\right)= \\
u_{Y} \otimes(+,-,-,+) \leftrightarrow\left(\frac{5}{2}, \frac{1}{2}, \frac{1}{2},-\frac{1}{2}\right)= \\
u_{Y} \otimes(+,+,-,-) \leftrightarrow\left(\frac{5}{2}, \frac{3}{2}, \frac{1}{2},-\frac{3}{2}\right) \times \\
u_{Y} \otimes(-,+,-,+) \leftrightarrow\left(\frac{3}{2}, \frac{3}{2}, \frac{1}{2},-\frac{1}{2}\right)= \\
u_{Y} \otimes(+,-,+,-)=\left(\frac{5}{2}, \frac{1}{2}, \frac{3}{2},-\frac{3}{2}\right) \times, \\
u_{Y} \otimes(-,-,+,+) \leftrightarrow\left(\frac{3}{2}, \frac{1}{2}, \frac{3}{2},-\frac{1}{2}\right) \times, \\
u_{Y} \otimes(-,+,+,-) \leftrightarrow\left(\frac{3}{2}, \frac{3}{2}, \frac{3}{2},-\frac{3}{2}\right)=\square \\
u_{Y} \otimes(-,-,-,-) \leftrightarrow\left(\frac{3}{2}, \frac{1}{2}, \frac{1}{2},-\frac{3}{2}\right)=\times .
\end{gathered}
$$


Then for $D_{4}$ we get. (We omit “ $V$ ".)

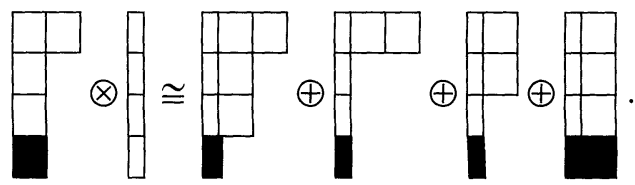

Hence,

$$
\begin{aligned}
& V\left(\Lambda_{1}+2 \Lambda_{3}\right) \otimes V_{\mathrm{sp}}^{(+)} \cong V\left(\Lambda_{1}+2 \Lambda_{3}+\Lambda_{4}\right) \oplus V\left(2 \Lambda_{1}+\Lambda_{3}\right) \\
& \oplus V\left(\Lambda_{2}+\Lambda_{3}\right) \oplus V\left(3 \Lambda_{3}\right) .
\end{aligned}
$$

\section{Decomposition of $V_{Y} \otimes V_{W}$}

6.1. In this section we treat general cases. Let $Y$ and $W$ be generalized Young diagrams of type $\mathrm{g}\left(=A_{n}, B_{n}, C_{n}, D_{n}\right)$. We shall give a combinatorial description for the irreducible decomposition of $V_{Y} \otimes V_{W}$. In Sects. 4 and 5, we treated special cases; $V_{W}$ is the vector representation or the spin representation. By the following lemma and the way of the construction of the crystal graph, we will know that these elementary cases play a significant role in general cases.

Lemma 6.1.1. Let $V_{j}(j=1, \ldots, p)$ be a finite dimensional irreducible representation of $U_{q}(\mathfrak{g})\left(\mathfrak{g}=A_{n}, B_{n}, C_{n}, D_{n}\right)$. For $u_{1} \otimes u_{2} \otimes \cdots \otimes u_{p} \in B\left(V_{1} \otimes \cdots \otimes V_{p}\right)$, the following two statements are equivalent;

(a) $u_{1} \otimes u_{2} \otimes \cdots \otimes u_{p}$ is a highest weight element of $B\left(V_{1} \otimes \cdots \otimes V_{p}\right)$.

(b) $u_{1} \otimes u_{2} \otimes \cdots \otimes u_{j}$ is a highest weight element of $B\left(V_{1} \otimes \cdots \otimes V_{j}\right)$ for any $j=1, \ldots, p$.

Proof. (a) follows trivially from (b). Next, we assume (a). For any $j$ we can consider $u_{1} \otimes u_{2} \otimes \cdots \otimes u_{p}=\left(u_{1} \otimes \cdots \otimes u_{j}\right) \otimes\left(u_{j+1} \otimes \cdots \otimes u_{p}\right) \in B\left(V_{1} \otimes \cdots \otimes V_{j}\right)$ $\otimes B\left(V_{j+1} \otimes \cdots V_{p}\right)$. By Proposition 3.2.1, if $\left(u_{1} \otimes \cdots \otimes u_{j}\right) \otimes\left(u_{j+1} \otimes \cdots \otimes u_{p}\right)$ satisfies the highest weight condition, $u_{1} \otimes \cdots \otimes u_{j}$ also satisfies the highest weight condition. Hence, we obtain (b). Q.E.D.

6.2. Remarks. Now, we give some remarks on the crystal graphs.

Remark 6.2.1.

(a) Let $W=\left(l_{1}, l_{2}, \ldots, l_{n}\right)$ be a generalized Young diagram of type $\mathfrak{g}\left(=A_{n}, B_{n}\right.$, $\left.C_{n}, D_{n}\right)$ with $l_{j} \in \mathbf{Z}$ for any $j$ and set $m:=l_{1}+\cdots+l_{n-1}+\left|l_{n}\right|$ ( $=$ the number of squares in $W)$. By the way of the construction of $B\left(V_{W}\right)$ in Sect. 2, any $u \in B\left(V_{W}\right)$ can be written in the following form,

$$
u=i_{1} \otimes i_{2} \otimes \cdots \otimes i_{m},
$$

where $i_{j}$ is an element of $B\left(V_{\square}\right)$.

(b) Let $W=\left(l_{1}, l_{2}, \ldots, l_{n}\right)$ be a generalized Young diagram of type $\mathrm{g}\left(\mathfrak{g}=B_{n}, D_{n}\right)$ with $l_{j} \in \mathbf{Z}+\frac{1}{2}$ for any $j$ and set $m:=\left(l_{1}-\frac{1}{2}\right)+\cdots+\left(l_{n-1}-\frac{1}{2}\right)+\left(\left|l_{n}\right|-\frac{1}{2}\right)$ ( = the number of squares in $W$ ). By the way of the construction of $B\left(V_{W}\right)$ in Sect. 2, any $u \in B\left(V_{W}\right)$ can be written in the following form,

$$
u=i_{1} \otimes i_{2} \otimes \cdots \otimes i_{m} \otimes v,
$$


where $i_{j}$ is an element of $B\left(V_{\square}\right)$ and $v$ is an element of $B_{\mathrm{sp}}$ or $B_{\mathrm{sp}}^{( \pm)}$.

6.3. Main theorem. Here, by Proposition 4.2.1, Proposition 5.1.2, Proposition 5.2.2, Lemma 6.1.1 and Remark 6.2.1, we obtain the following theorem.

Theorem 6.3.1. (1) Let $W$ and $m$ be as in Remark 6.2.1(a) and $Y$ a generalized Young diagram of type $\mathrm{g}\left(=A_{n}, B_{n}, C_{n}, D_{n}\right)$. Then we obtain

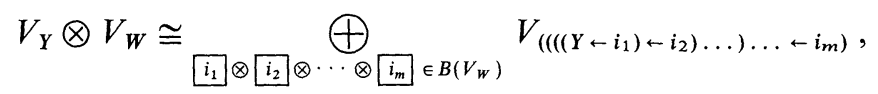

where $V_{\left(\left(\left(\left(Y \leftarrow i_{1}\right) \leftarrow i_{2}\right) \ldots\right) \ldots \leftarrow i_{m}\right)}$ is the 0 -dimensional vector space if there exists $k \in\{1, \ldots, m\}$ such that $\left(\left(\left(\left(Y \leftarrow i_{1}\right) \leftarrow i_{2}\right) \ldots\right) \ldots \leftarrow i_{k}\right)$ is not a generalized Young diagram of type $\mathrm{g}$.

(2) Let $W$ and $m$ be as in Remark 6.2.1(b) and $Y$ a generalized Young diagram of type $\mathfrak{g}\left(=B_{n}, D_{n}\right)$. Then we obtain

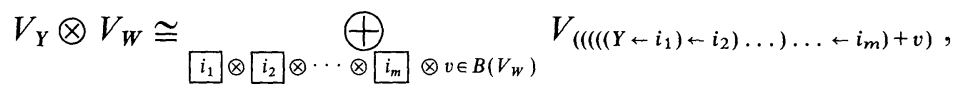

where $V_{\left.\left.\left(\left(\left(()+i_{1}\right) \leftarrow i_{2}\right) \ldots\right) \ldots \leftarrow i_{m}\right)+v\right)}$ is the 0 -dimensional vector space if there exists $k \in\{1, \ldots, m\}$ such that $\left(\left(\left(\left(Y \leftarrow i_{1}\right) \leftarrow i_{2}\right) \ldots\right) \ldots \leftarrow i_{k}\right)$ is not a generalized Young diagram of type $\mathrm{g}$ or $\left(\left(\left(\left(\left(Y \leftarrow i_{1}\right) \leftarrow i_{2}\right) \ldots\right) \ldots \leftarrow i_{m}\right)+v\right)$ is not a generalized Young diagram of type $\mathrm{g}$.

Corollary 6.3.2. Let $J=\{1,2, \ldots, p\}$ be a finite index set and $Y$ and $W_{j}(j \in J)$ be generalized Young diagrams of type g. We obtain

$$
V_{Y} \otimes V_{W_{1}} \otimes \cdots \otimes V_{W_{p}} \cong \bigoplus_{u_{j} \in B\left(V_{w_{j}}\right)(j \in J)} V_{\left(\left(\left(\left(Y \leftarrow u_{1}\right) \Leftarrow u_{2}\right) \Leftarrow \ldots\right) \ldots \Leftarrow u_{p}\right)},
$$

where for $Y$ and $u_{j} \in B\left(V_{W_{j}}\right)$, if $u_{j}$ is in the form (6.2.1), we define

$$
\left(Y \leftarrow u_{j}\right):=\left(\left(\left(\left(Y \leftarrow i_{1}\right) \leftarrow i_{2}\right) \ldots\right) \ldots \leftarrow i_{m}\right),
$$

and if $u_{j}$ is in the form (6.2.2), we define

$$
\left(Y \Leftarrow u_{j}\right):=\left(\left(\left(\left(\left(Y \leftarrow i_{1}\right) \leftarrow i_{2}\right) \ldots\right) \ldots \leftarrow i_{m}\right)+v\right)
$$

and $V_{\left(\left(\left(\left(Y \leftarrow u_{1}\right) \Leftarrow u_{2}\right) \leftarrow \ldots\right) \ldots \Leftarrow u_{p}\right)}$ is the 0 -dimensional vector space if there exists $j \in J$ such that $\left(\left(\left(\left(Y \Leftarrow u_{1}\right) \Leftarrow u_{2}\right) \Leftarrow \cdots\right) \cdots \Leftarrow u_{j}\right)$ is not a generalized Young diagram of type $\mathfrak{g}$.

Example 6.3.3. For $\mathfrak{g}=\mathfrak{g l}(3), Y=\square$ and $W=\square$, we consider $V_{Y} \otimes V_{W}$.

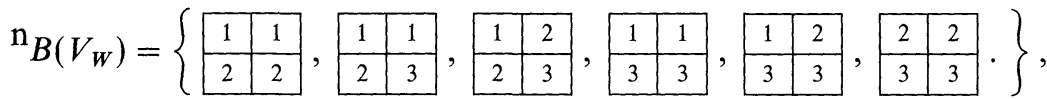

$$
\begin{aligned}
& u_{Y} \otimes \begin{array}{|l|l|}
\hline 1 & 1 \\
\hline 2 & 2 \\
\hline
\end{array}((((\square \leftarrow 1) \leftarrow 2) \leftarrow 1) \leftarrow 2)=\square \square \square, \\
& u_{Y} \otimes \begin{array}{|l|l}
\hline 1 & 1 \\
\hline 2 & 3
\end{array} \leftrightarrow((((\square \square+1) \leftarrow 3) \leftarrow 1) \leftarrow 2)=\square, \\
& u_{Y} \otimes \begin{array}{|l|l}
\hline 1 & 2 \\
\hline 2 & 3
\end{array} \leftrightarrow((((\square \leftarrow 2) \leftarrow 3) \leftarrow 1) \leftarrow 2)=\square,
\end{aligned}
$$




$$
\begin{aligned}
& u_{Y} \otimes \begin{array}{|l|l|}
\hline 1 & 1 \\
\hline 3 & 3
\end{array} \leftrightarrow((((\square \leftarrow 1) \leftarrow 3) \leftarrow 1) \leftarrow 3) \times, \\
& u_{Y} \otimes \begin{array}{|l|l|}
\hline 1 & 2 \\
\hline 3 & 3
\end{array} \leftrightarrow((((\square \square \leftarrow 2) \leftarrow 3) \leftarrow 1) \leftarrow 3)=(\square, \\
& \hline \square
\end{aligned}
$$

Hence by Theorem 6.3.1.(1), for gl(3) we obtain (we omit " $V$ ")

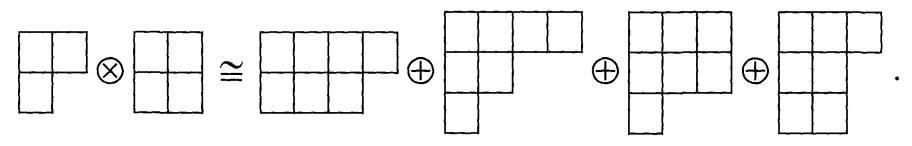

Example 6.3.4. For $\mathrm{g}=B_{2}$, we consider $V\left(4 \Lambda_{2}\right) \otimes V\left(\Lambda_{1}+\Lambda_{2}\right)$, where

$$
4 \Lambda_{2} \Leftrightarrow(2,2)=\square, \quad \Lambda_{1}+\Lambda_{2} \Leftrightarrow\left(\frac{3}{2}, \frac{1}{2}\right)=\square .
$$

We set $Y=\square$ and $W=\square$. By Theorem 2.3 .3 (ii), we have

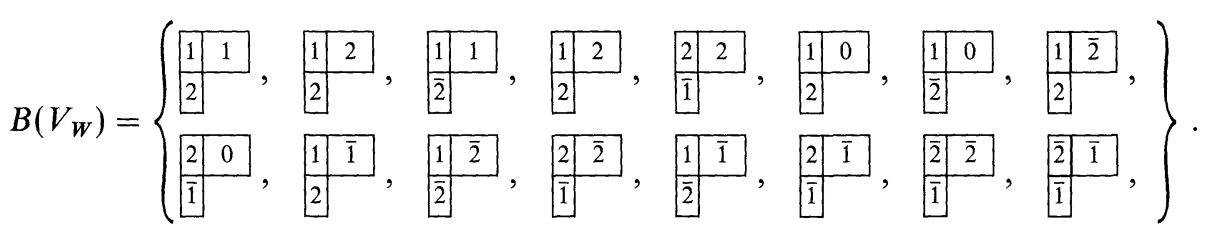

\section{Hence,}

$$
\begin{aligned}
& \left.u_{Y} \otimes \frac{1}{2} 1\right]=u_{Y} \otimes \square \otimes(+,+) \leftrightarrow((Y \leftarrow 1)+(+,+))=\left(\frac{7}{2}, \frac{5}{2}\right)=\square \square,
\end{aligned}
$$

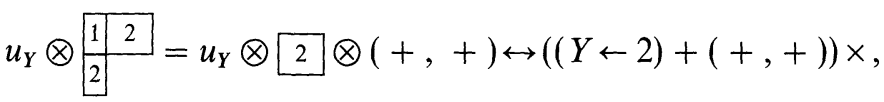

$$
\begin{aligned}
& u_{Y} \otimes \frac{1}{2}=u_{Y} \otimes \square \otimes(+,-) \leftrightarrow((Y \leftarrow 1)+(+,-))=\left(\frac{7}{2}, \frac{3}{2}\right)=\square,
\end{aligned}
$$

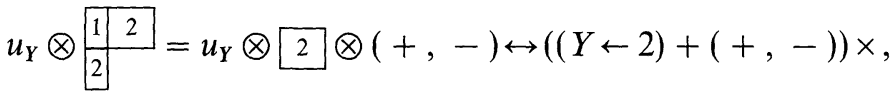

$$
\begin{aligned}
& u_{Y} \otimes \begin{array}{l|l}
2 & 2 \\
\hline 1 & =u_{Y} \otimes 2
\end{array}(-,+) \leftrightarrow((Y \leftarrow 2)+(-,+)) \times, \\
& u_{Y} \otimes \begin{array}{l|l}
1 & 0 \\
\hline 2
\end{array}=u_{Y} \otimes 0 \otimes(+,+) \leftrightarrow((Y \leftarrow 0)+(+,+))=\left(\frac{5}{2}, \frac{5}{2}\right)=\square \square
\end{aligned}
$$




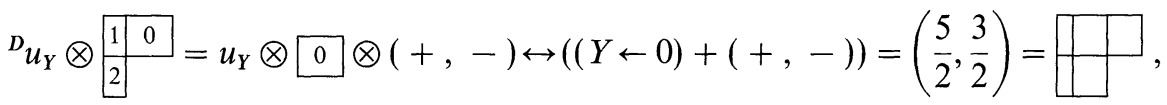

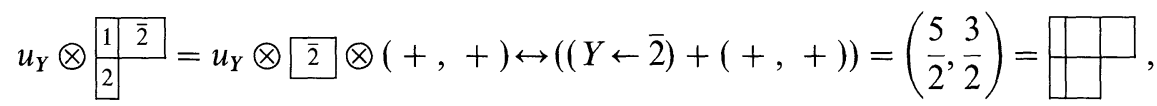

$$
\begin{aligned}
& u_{Y} \otimes \begin{array}{|l|l}
\hline 2 & 0 \\
\hline \overline{1} & =u_{Y} \otimes 0
\end{array} \otimes(-,+) \leftrightarrow((Y \leftarrow 0)+(-,+))=\times,
\end{aligned}
$$

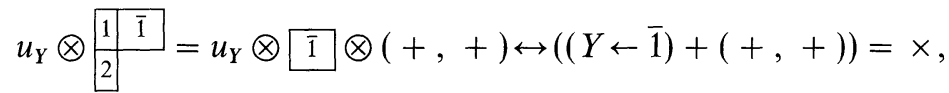

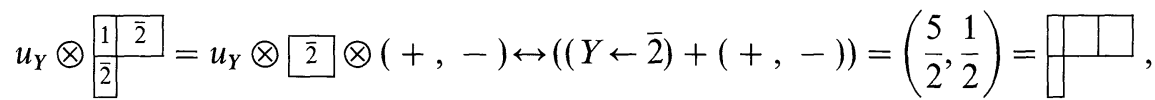

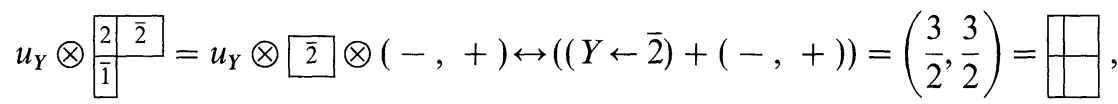

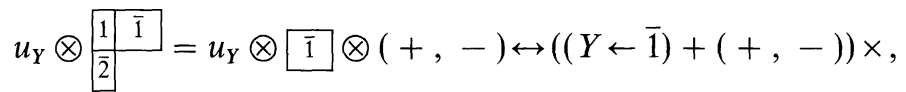

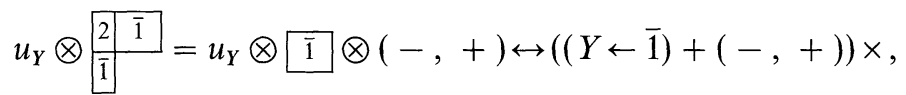

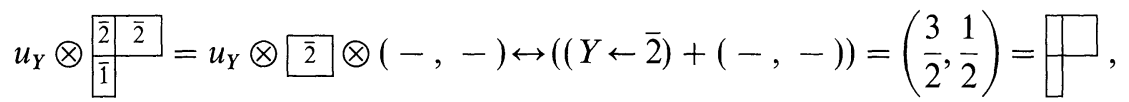

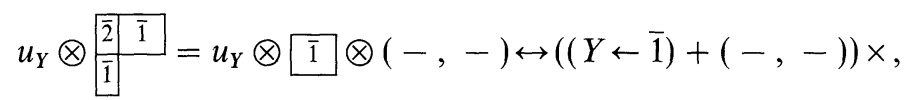

By Theorem 6.3.1.(2), for $B_{2}$ we obtain (we omit " $V$ ").

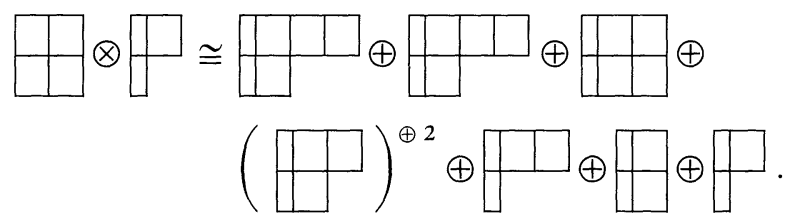

Hence,

$$
\begin{gathered}
V\left(4 \Lambda_{2}\right) \otimes V\left(\Lambda_{1}+\Lambda_{2}\right) \cong V\left(\Lambda_{1}+5 \Lambda_{2}\right) \oplus V\left(2 \Lambda_{1}+3 \Lambda_{2}\right) \oplus V\left(5 \Lambda_{2}\right) \\
\oplus V\left(\Lambda_{1}+3 \Lambda_{2}\right)^{\oplus 2} \oplus V\left(2 \Lambda_{1}+\Lambda_{2}\right) \oplus V\left(3 \Lambda_{2}\right) \oplus V\left(\Lambda_{1}+\Lambda_{2}\right) .
\end{gathered}
$$

\section{Appendix. Relation to the Original Littlewood-Richardson Rule}

The original description of the Littlewood-Richardson rule for the $\mathfrak{g}=\mathfrak{g l}(n+1)$ case is different from ours. In this appendix, we shall give their relation. This relation is well-known to the specialists (e.g. cf. [W]).

First, we explain the original Littlewood-Richardson rule ([M]). 
For Young diagrams $Y=\left(l_{1}, \ldots, l_{n+1}\right)$ and $Z=\left(m_{1}, \ldots, m_{n+1}\right), Y \geqq Z$ if $l_{j} \geqq m_{j}$ for any $j$. If $Y \geqq Z$, the set-theoretic difference $W=Y-Z$ is called a skew diagram.

Definition A. Let $J=\{1,2, \ldots, n+1\}$ be a finite index set. A finite sequence $s_{1} s_{2} \cdots s_{p}\left(s_{j} \in J\right)$ is said to be a lattice permutation if the following condition is satisfied;

$$
\begin{aligned}
& \#\left\{k \mid s_{k}=i \text { and } 1 \leqq k \leqq r\right\} \geqq \#\left\{k \mid s_{k}=i+1 \text { and } 1 \leqq k \leqq r\right\} \\
& \qquad \text { for any } 1 \leqq i \leqq n \text { and } 1 \leqq r \leqq p .
\end{aligned}
$$

Let $W=Y-Z$ be a skew diagram and $T_{W}$ a semi-standard skew tableau of shape $W$ with symbols $J$ (see [M]). For a Young diagram $Y^{\prime}=\left(l_{1}^{\prime}, \ldots, l_{n+1}^{\prime}\right)$, we say that $w t\left(T_{W}\right)=w t\left(Y^{\prime}\right)$ if the number of symbols $j$ in $W$ is equal to $l_{j}^{\prime}$ for any $j$. From $T_{W}$ we derive a sequence $s\left(T_{W}\right)$ by reading the symbols in $T_{W}$ from the right to the left in successive row.

Theorem B (the Littlewood-Richardson rule). Let $V_{Y}$ and $V_{W}$ be irreducible $\mathrm{gl}(n+1)$-modules associated with Young diagrams $Y$ and $W$ with $n+1$ rows. Then we obtain

$$
V_{Y} \otimes V_{W} \cong \underset{\substack{T_{Z-Y} \text { is semi-standard, } \\ w t\left(T_{Z-\gamma}=w(W) \text { and } \\ s\left(T_{Z}-\gamma\right)\right. \text { is a lattice permutation. }}}{\bigoplus} V_{Z} .
$$

For Young diagrams $Y, W$ and $Z$ in $(*)$, let us define the map $\Phi$ which associates a semi-standard skew tableau $T_{Z-Y}$ as in $(*)$ with a semi-standard tableau of shape $W$.

$\Phi$ : If there is a symbol $k(1 \leqq k \leqq n+1)$ in the $m$-th row $(1 \leqq m \leqq n+1)$ in $T_{Z-Y}$, then a symbol $m$ is written in the $k$-th row in the diagram $W$.

Theorem C. For Young diagrams $Y$ and $W$, we set

$$
\begin{aligned}
& \mathscr{T}(Y, W)=\left\{T_{Z-Y} ; \begin{array}{l}
Z \geqq Y, w t\left(T_{Z-Y}\right)=w t(W) \text { and }, \\
s\left(T_{Z-Y}\right) \text { is a lattice permutation. } .
\end{array}\right\}, \\
& B^{h}(Y, W)=\left\{b \in B\left(V_{W}\right) ; u_{Y} \otimes b \text { is a highest weight element of } B\left(V_{Y} \otimes V_{W}\right)\right\} .
\end{aligned}
$$

Then $\Phi$ gives a $1-1$ correspondence between $\mathscr{T}(Y, W)$ and $B^{h}(Y, W)$.

Example D. For $\mathfrak{g}=\mathfrak{g l}(3), Y=(2,1,0)=\square$ and $W=(2,2,0)=\square$, which is introduced in Sect. 6, we have

$$
\begin{aligned}
& \mathscr{T}(Y, W)=\left\{\begin{array}{|l|l|l|l|}
\hline & & 1 & 1 \\
\hline & 2 & 2 \\
\hline
\end{array}, \begin{array}{|l|l|l|l|}
\hline & & 1 & 1 \\
\hline & 2 & \\
\hline 2 &
\end{array}, \begin{array}{|l|l|l|l|l|l|}
\hline & & 1 \\
\hline & 1 & 2 \\
\hline 2 &
\end{array}, \begin{array}{|l|l|l|}
\hline & & 1 \\
\hline
\end{array}\right\}, \\
& B^{h}(Y, W)=\left\{\begin{array}{|l|l|}
\hline 1 & 1 \\
\hline 2 & 2 \\
\hline
\end{array}, \begin{array}{|l|l|}
\hline 1 & 1 \\
\hline 2 & 3 \\
\hline
\end{array}, \begin{array}{|l|l|}
\hline 1 & 2 \\
\hline 2 & 3 \\
\hline
\end{array}, \begin{array}{|l|l|}
\hline 1 & 2 \\
\hline 3 & 3 \\
\hline
\end{array}\right\} .
\end{aligned}
$$


Hence, we obtain

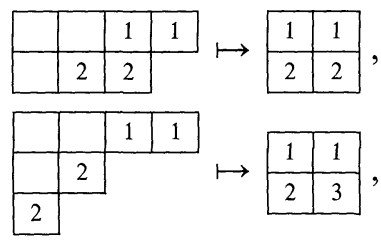

$\Phi:$

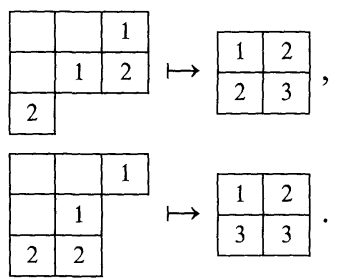

$k$ in the $m$-th row $\mapsto m$ in the $k$-th row

\section{References}

[B-Z] Berenstein, A.D., Zelevinski, A.V.: Tensor product multiplicities and convex polytopes in partition space. J. Geom. Phys. 5, 453-472 (1989)

[D] Drinfeld, V.G.: Quantum Groups. ICM proceedings, Berkely, 798-820 (1986)

[J] Jimbo, M.: A $q$-difference analogue of $U(\mathrm{~g})$ and the Yang-Baxter equation. Lett. Math. Phys. 11, 247-252 (1986)

[K1] Kashiwara, M.: Crystallizing the $q$-analogue of universal enveloping algebra. Commun. Math. Phys. 133, 249-260 (1990)

[K2] Kashiwara, M.: On crystal base of $q$-analogue of universal enveloping algebras. Duke. Math. J. 63, 465-516 (1991)

[K-N] Kashiwara, M., Nakashima, T.: Crystal graphs for Representations of the $q$-analogue of Classical Lie Algebras. RIMS preprint, 767 (1991)

[L] Littelmann, P.: A Generalization of the Littlewood-Richardson Rule. J. Algebra 130, 328-368 (1990)

[M] Macdonald, I.G.: Symmetric Functions and Hall Polynomials. Oxford: Clarendon Press, 1979

[N] Nakashima, T.: A basis of symmetric tensor representations for the quantum analogue of the Lie algebra $B_{n}, C_{n}$ and $D_{n}$. Publ. RIMS. Kyoto. Univ. 26, 723-733 (1990).

[O] Okado, M.: Quantum $R$ Matrices Related to the spin Representations of $B_{n}$ and $D_{n}$. Commun. Math. Phys. 134, 467-486 (1990)

[Re] Reshetikhin, N.Yu.: Quantized universal enveloping algebras. Yang-Baxter equation and invariants of links I. LOMI preprint E-4-48 (1990)

[T] Thomas, G.: On Schensted's construction and the multiplication of Schur function. Adv. Math. 30, 8-32 (1978)

[W] Weyman, J.: Pieri's formulas for classical groups. Contemp. Math. 88, 177-184 (1989)

Communicated by N.Yu. Reshetikhin 
